\title{
Exploring degrees of entanglement
}

\section{Citation}

Myers, John M., and Tai Tsun Wu. 2009. "Exploring Degrees of Entanglement." Quantum Information Processing 9 (2) (October 16): 239-271. doi:10.1007/s11128-009-0146-5.

\section{Published Version}

doi:10.1007/s11128-009-0146-5

\section{Permanent link}

http://nrs.harvard.edu/urn-3:HUL.InstRepos:34903211

\section{Terms of Use}

This article was downloaded from Harvard University's DASH repository, and is made available under the terms and conditions applicable to Open Access Policy Articles, as set forth at http:// nrs.harvard.edu/urn-3:HUL.InstRepos:dash.current.terms-of-use\#OAP

\section{Share Your Story}

The Harvard community has made this article openly available.

Please share how this access benefits you. Submit a story.

\section{Accessibility}




\title{
Exploring Degrees of Entanglement
}

\author{
John M. Myers and Tai Tsun Wu \\ School of Engineering and Applied Sciences, \\ Harvard University, Cambridge, MA 02138, USA
}

In spite of a long history, the quantification of entanglement still calls for exploration. What matters about entanglement depends on the situation, and so presumably do the numbers suitable for its quantification. Regardless of situational complications, a necessary first step is to make available for calculation some quantitative measure of entanglement. Here we define a geometric degree of entanglement, distinct from earlier definitions, but in the case of bipartite pure states related to that proposed by Shimony (1995). The definition offered here applies also to multipartite mixed states, and a variational method simplifies the calculation.

We analyze especially states that are invariant under permutation of particles, states that we call bosonic. Of interest to quantum sensing, for bosonic states, we show that no partial trace can increase a degree of entanglement. For some sample cases we quantify the degree of entanglement surviving a partial trace. As a function of the degree of entanglement of a bosonic 3-qubit pure state, we show the range of degree of entanglement for the 2-qubit reduced density matrix obtained from it by a partial trace. Then we calculate the degree of entanglement of the mixed state obtained as a partial trace over one qubit of a 4-qubit bosonic state.

As a reminder of the situational dependence of the advantage of entanglement, we review the way in which entanglement combines with scattering theory in the example of lightbased radar.

\section{INTRODUCTION}

As early as Born's 1926 analysis of scattering [1], the correlations peculiar to quantum physics were noticed, and since then have been exploited in applications including cryptography. Yet the investigation of quantitative measures of entanglement is still in a stage that can only be called "exploratory." What matters about entanglement depends on the situation, and so presumably do the numbers suitable for its quantification. As an example of situation dependence, light-based radar (ladar) stands to benefit from the use of entangled quantum states, and in this application the loss of particles between transmission and reception needs to be accounted for in assessing possible benefits of entanglement.

Regardless of situational complications, a necessary first step is to make available some quantitative measure of entanglement. In the next section we define a geometric degree of entanglement, distinct from earlier definitions, but in the case of bipartite pure states related to that of Shimony [2]. The definition offered here extends beyond the bipartite case to cover multipartite mixed states. Being a definition, its justification must lie in what it leads to [3]. 
The problem arises of calculating the degree of entanglement for given pure and mixed states. After outlining in Sec. III some techniques, we turn in Sec. IV to the calculation of some cases of pure-state degrees of entanglement, and in Sec. V to the calculation of mixed-state degrees of entanglement. As with other measures of entanglement, one encounters the obstacle that for mixed states the calculation, to put it mildly, is challenging. We make use of a variational method to simplify greatly the calculation of the degree of entanglement of mixed states. Many of the cases of pure and mixed states addressed in this paper are for states that are invariant under permutation of particles, states that we call bosonic. These offer the charm of low dimensional vector spaces as a wedge into a complicated subject, along with other advantages noted below.

Equipped with a calculable, quantitative measure of entanglement, lots of questions can be asked. With an eye toward quantum ladar, in Sec. VI we begin to study the degree of entanglement that survives a partial trace that expresses a loss of some particles. For states without special symmetry, nothing general can be said in this connection; however for bosonic states we show that no partial trace ever increases a degree of entanglement. We also show examples of numerical relations among degrees of entanglement of a 4-qubit pure symmetric state and degrees of entanglement of its partial traces over one and two qubits.

As a reminder of the situational dependence of the advantage of entanglement, we review in Sec. VII the way in which entanglement combines with scattering theory in connection with ladar in what is called Type-III sensing, in which part of the signal generated by a transmitter is stored for later comparison with the echo from the part of the signal incident on a target.

\section{DEGREE OF ENTANGLEMENT}

To build up a general definition of the degree of entanglement used in this paper, we start with the case of bipartite pure states. Familiar in the literature is the geometric measure of the entanglement of a normalized bipartite pure state $|\hat{\psi}\rangle$ as the distance between the vector $|\hat{\psi}\rangle$ and the nearest unentangled normalized vector [2]. By dropping the requirement that the nearest unentangled vector be normalized, we define a degree of entanglement applicable not just to the normalized state $|\hat{\psi}\rangle$ but also to unnormalized states, which simplifies the analysis to come.

Consider two particles designated 1 and 2 (corresponding to states in complex vector spaces $\mathcal{H}_{1}$ and $\mathcal{H}_{2}$, respectively. Let $N_{i}=\operatorname{dim}_{\mathbb{C}}\left(\mathcal{H}_{i}\right), i=1,2$. Denote states of an orthonormal basis of $\mathcal{H}_{1}$ by $|k\rangle, k=1, \ldots, N_{1}$, and denote states of an orthonormal basis of $\mathcal{H}_{2}$ by $|\ell\rangle, \ell=1, \ldots, N_{2}$.

We define the degree of entanglement of a bipartite pure state, whether it is normalized or not, as follows. The general bipartite pure state for the particles 1 and 2 taken together is a vector in the tensor-product space $\mathcal{H}_{1} \otimes \mathcal{H}_{2}$ :

$$
|\psi\rangle=\sum_{k=1}^{N_{1}} \sum_{\ell=1}^{N_{2}} \psi_{k l}|k, \ell\rangle,
$$

from which it follows immediately that

$$
\langle\psi \mid \psi\rangle=\sum_{k=1}^{N_{1}} \sum_{\ell=1}^{N_{2}}\left|\psi_{k l}\right|^{2} .
$$

Define the set of unentangled pure states (meaning pure states that are tensor products) to be

$$
\left.\Phi=\left\{|\phi\rangle\left|\left(\exists x_{k}, y_{\ell}\right)\right| \phi\right\rangle=\left(\sum_{k=1}^{N_{1}} x_{k}|k\rangle\right)\left(\sum_{\ell=1}^{N_{2}} y_{\ell}|\ell\rangle\right)\right\} .
$$


As noted above, this set includes unnormalized unentangled states. We define the degree of entanglement $D(|\psi\rangle)$ of an arbitrary vector $|\psi\rangle$ by

$$
D(|\psi\rangle) \stackrel{\text { def }}{=} \min _{|\phi\rangle \in \Phi} \||\psi\rangle-|\phi\rangle \| .
$$

As shown in Appendix A, this degree of entanglement of a state $|\psi\rangle$ that need not be normalized can also be expressed by its projection onto the nearest normalized, unentangled state; that is the square of the degree of entanglement satisfies

$$
[D(|\psi\rangle)]^{2}=\langle\psi \mid \psi\rangle-\max _{|\hat{\phi}\rangle}|\langle\psi \mid \hat{\phi}\rangle|^{2},
$$

where the maximum is over all unentangled states $|\hat{\phi}\rangle$ normalized so that $\langle\hat{\phi} \mid \hat{\phi}\rangle=1$.

For any positive matrix $A$, let $\Lambda_{\max }(A)$ denote the largest eigenvalue of $A$. Also shown in Appendix A is the result

$$
[D(|\psi\rangle)]^{2}=\langle\psi \mid \psi\rangle-\Lambda_{\max }\left[\operatorname{tr}_{2}(|\psi\rangle\langle\psi|)\right],
$$

where $\operatorname{tr}_{2}(|\psi\rangle\langle\psi|)$ is the partial trace over $\mathcal{H}_{2}$, which can be called a generalized reduced density matrix. By a generalized density matrix we mean a positive matrix, without requiring unit trace; in case $\langle\psi \mid \psi\rangle=1$ then $\Lambda_{\max }\left[\operatorname{tr}_{2}(|\psi\rangle\langle\psi|)\right]$ is the largest eigenvalue of the reduced density matrix $[4,5]$. Because the eigenvalues of reduced density matrices (and also generalized reduced density matrices) are the same for tracing over $\mathcal{H}_{1}$ as for tracing over $\mathcal{H}_{2}$ [6], one gets the same result if in Eq. (6) $\operatorname{tr}_{2}$ is replaced by $\operatorname{tr}_{1}$.

\section{A. Degrees of entanglement for multipartite states}

Consider pure states on a Hilbert space $\mathcal{H}=\mathcal{H}_{1} \otimes \mathcal{H}_{2} \otimes H_{3} \ldots \mathcal{H}_{n}$. A vector in $\mathcal{H}$ has components indexed by $n$ indices, that is, by as many indices as there are factor spaces of $\mathcal{H}$. Any partition of these indices into two blocks, denoted I and II, allows us to view $\mathcal{H}$ as a bipartite product $\mathcal{H} \cong \mathcal{H}_{\mathrm{I}} \otimes \mathcal{H}_{\mathrm{II}}$, so that one can define what we call a split degree of entanglement for a multipartite pure state $|\psi\rangle \in \mathcal{H}$ as in the bipartite case, namely as

$$
D_{\mathrm{I} \mid \mathrm{II}}(|\phi\rangle)=\min _{\left|\phi_{\mathrm{I}}\right\rangle \in \mathcal{H}_{\mathrm{I}},\left|\phi_{\mathrm{II}}\right\rangle \in \mathcal{H}_{\mathrm{II}}} \||\phi\rangle-\left|\phi_{\mathrm{I}}\right\rangle\left|\phi_{\mathrm{II}}\right\rangle \| .
$$

For $|\psi\rangle$ a multipartite vector, we define its degree of entanglement $D(|\psi\rangle)$ to be the minimum over all partitions of its indices of the split degrees of entanglement.

For example, any 3-qubit pure state $|\psi\rangle \in \mathcal{H}_{1} \otimes \mathcal{H}_{2} \otimes \mathcal{H}_{3}$ has the form

$$
|\psi\rangle=\sum_{j=0}^{1} \sum_{k=0}^{1} \sum_{\ell=0}^{1} \psi_{j, k, \ell}|j\rangle|k\rangle|\ell\rangle .
$$

Three splittings of $\mathcal{H}_{1} \otimes \mathcal{H}_{2} \otimes \mathcal{H}_{3}$ lead to three split degrees of entanglement $D_{1 \mid 23}(|\psi\rangle), D_{2 \mid 13}(|\psi\rangle)$, and $D_{3 \mid 12}(|\psi\rangle)$, so we have

$$
D(|\psi\rangle)=\min \left\{D_{1 \mid 23}(|\psi\rangle), D_{2 \mid 13}(|\psi\rangle), D_{3 \mid 12}(|\psi\rangle)\right\} .
$$


Question: are there any 3-qubit pure states that have maximal degree of entanglement for all three splittings? Answer: yes; indeed Sec. IV displays a manifold of such states.

The bad news is that as the number of tensor-product factors increases, the number of splits rises rapidly. For a $k$-fold multipartite space $\mathcal{H}$ there are

$$
\frac{1}{2} \sum_{\ell=1}^{k-1}\left(\begin{array}{l}
k \\
\ell
\end{array}\right)=2^{n-1}-1
$$

distinct splits. While no conceptual problem, this large number is another motivation to study states that are symmetric under exchange of particles, for in the symmetric case the number of splits that need to be calculated for a $k$-fold multipartite space drops to the greatest integer less than or equal to $k / 2$.

\section{B. Degree of entanglement for mixed states}

Now for mixed states. Any mixed state $\rho$ has many decompositions into weighted sums over unnormalized pure-state density matrices

$$
\rho=\sum_{i}\left|\psi_{i}\right\rangle\left\langle\psi_{i}\right|, \quad \sum_{i}\left\langle\psi_{i} \mid \psi_{i}\right\rangle=1
$$

Designating an arbitrary split degree of entanglement by $D_{\text {split }}$, we define $D_{\text {split }}(\rho)$ in terms of the minimum over all such decompositions by

$$
\begin{aligned}
D_{\text {split }}^{2}(\rho) & \stackrel{\text { def }}{=} \min _{\text {decomp. }} \sum_{i} D_{\text {split }}^{2}\left(\left|\psi_{i}\right\rangle\right), \\
D(\rho) & \stackrel{\text { def }}{=} \min _{\text {splits }} D_{\text {split }}(\rho) .
\end{aligned}
$$

For $\rho$ any 2-qubit state (but only this case) it is easy to check that

$$
D^{2}(\rho)=\frac{1}{2}\left(1-\sqrt{1-C^{2}(\rho)}\right),
$$

where $C(\rho)$, defined only for two-qubit matrices, is Wootters' concurrence [7].

If one prefers to use normalized state $\left|\hat{\psi}_{i}\right\rangle$ in decompositions in place of the unnormalized states $\left|\psi_{i}\right\rangle$ used here, one has only to note that $D^{2}\left(\left|\psi_{i}\right\rangle\right)=\left\langle\psi_{i} \mid \psi\right\rangle D^{2}\left(\left|\hat{\psi}_{i}\right\rangle\right)$ where the normalized state $\left|\hat{\psi}_{i}\right\rangle=\left|\psi_{i}\right\rangle / \sqrt{\left\langle\psi_{i} \mid \psi\right\rangle}$.

In common with entanglement measures discussed in [4], $D$ exhibits the properties

$$
\begin{aligned}
D(\rho) & \geq 0, \\
D(\rho) & =0 \quad \text { if } \rho \text { is separable, } \\
\sum_{i} p_{i} D^{2}\left(\rho_{i}\right) & \geq D^{2}\left(\sum_{i} p_{i} \rho_{i}\right)
\end{aligned}
$$

as well as the fact that $D^{2}(\rho)=D^{2}\left(U \rho U^{\dagger}\right)$ whenever $U$ belongs to the group of local unitary transformations.

For density matrices $\rho$ as operators on $\mathcal{H}^{\otimes n}$, for which all factor spaces have the same dimension, we also have that $\left(\forall s \in S_{n}\right) D\left(s \rho s^{\dagger}\right)=D(\rho)$ where $S_{n}$ is the group of permutations of factor spaces spelled out in Appendix Appendix B B.3.

In Sec. $\mathrm{V}$ we apply a variational technique to replace the minimum over decompositions with equations that define the split degrees of entanglement. 


\section{TECHNIQUES}

\section{A. Invariance of degree of entanglement under local unitary transformations}

We make use of group actions on pure states, and these extend to actions on mixed states. For example, any group $G$ that acts on a Hilbert space $\mathcal{H}=\mathcal{H}_{1} \otimes \mathcal{H}_{2} \otimes \mathcal{H}_{3} \otimes \ldots$ partitions $\mathcal{H}$ into orbits. In the cases at hand, the quotient map of $\mathcal{H}$ onto the set of orbits makes a space of orbits. Because the degrees of entanglement are all invariant under transformations belonging to "local" unitary groups $U(1) \otimes S U\left(N_{1}\right) \otimes S U\left(N_{2}\right) \otimes S U\left(N_{3}\right) \otimes \ldots$ that act separately on the factor spaces $\mathcal{H}_{j}$ of $\mathcal{H}$ (where $\operatorname{dim}_{\mathbb{C}} \mathcal{H}_{j}=N_{j}$ ), any degree of entanglement can be thought of as a function on a space of orbits. (Note that in defining the "local" groups we choose to include the phase factor $U(1)$.) When studying bosonic states - invariant under permutation of factors (see Appendix Appendix B B.3) - we replace local groups by subgroups that preserve bosonic symmetry.

In the next sections we demonstrate several relations pertaining to degrees of entanglement of various pure and mixed states as follows:

1. Specify a space of quantum states and a local transformation group $G$ that acts on this space.

2. Simplify analysis by making adroit choices of representative states of all the $G$-orbits.

3. Demonstrate that a relation holds for the chosen representative states.

4. Invoke the invariance of the degree of entanglement under $G$ to conclude that the property holds not only for the chosen representative but for the orbit space (and hence for all the states).

An example of representative states for 3-qubit orbits is in Acin et al. [8].

\section{B. Bosonic symmetry}

As a wedge into the complicated topic of quantifying entanglement, states that are invariant under permutation of particles offer attractive simplifications. We call such states, whether pure or mixed, bosonic. In order for the pure or mixed states associated with a tensor-product Hilbert space $\mathcal{H}=\mathcal{H}_{1} \otimes \mathcal{H}_{2} \otimes \mathcal{H}_{3} \otimes \ldots \otimes \mathcal{H}_{n}$ to be symmetric under exchange of particles, all the factor spaces must have the same dimension, so the vector space of $n$ indistinguishable particles, each with $N$ levels, is a subspace of

$$
\mathcal{H}=\mathcal{H}^{\otimes n} .
$$

While $\operatorname{dim}_{\mathbb{C}} \mathcal{H}^{\otimes n}=N^{n}$, the bosonic subspace has for its complex dimension just the number of ways that $n$ indistinguishable particles can be distributed into $N$ bins, which is

$$
\left(\begin{array}{c}
n+N-1 \\
n
\end{array}\right) \stackrel{\text { def }}{=} \frac{(n+N-1) !}{n !(N-1) !} .
$$

For $N>1$, the dimension stated in (17) drops exponentially below $N^{n}$ as the number of particles $n$ grows. For a multiqubit state, $N=2$ and the complex dimension of an $n$-qubit vector space of states symmetric under particle interchange is $n+1$ in contrast to $2^{n}$ when no symmetry constraint is imposed.

The group of local unitary transformations of $\mathcal{H}^{\otimes n}$ is $U(1) \otimes S U(N)^{\otimes n}$, where $N=\operatorname{dim}_{\mathbb{C}} \mathcal{H}$. When bosonic symmetry is imposed, one employs the corresponding subgroup of local unitary 
transformations that preserve symmetry under particle exchange. As discussed explicitly below for the case of qubit spaces, this "local bosonic group" is isomorphic to $U(N)$. When the single particle is a qubit, the "local bosonic group" is isomorphic to $U(2)$.

\section{Remarks on dimensions of orbits and orbit spaces}

Recall that symmetry conditions give information about the dimensions of orbits and orbit spaces associated with actions of a Lie group on a manifold. Both real and complex dimensions enter the story, and we say which we mean on each occasion. For a Lie group $G$ acting on a manifold $X$, for any point $x \in X$ we have that for the dimension of the orbit $G x$

$$
\operatorname{dim}_{\mathbb{R}} G x=\operatorname{dim}_{\mathbb{R}} G-\operatorname{dim}_{\mathbb{R}} G_{x},
$$

where $G_{x}$ is the stabilizer subgroup of $G$, the subgroup that leaves $x$ unaffected. The dimension of the space of orbits is then

$$
\operatorname{dim}_{\mathbb{R}} X-\operatorname{dim}_{\mathbb{R}} G+\operatorname{dim}_{\mathbb{R}} \text { (generic stabilizer subgroup). }
$$

The most general space of interest here is the manifold of normalized vectors in the general tensorproduct space $\mathcal{H}=\mathcal{H}_{1} \otimes \mathcal{H}_{2} \otimes \mathcal{H}_{3} \otimes \ldots \otimes \mathcal{H}_{n}$ under the action of the local unitary group $G \stackrel{\text { def }}{=} U(1) \otimes S U\left(N_{1}\right) \otimes S U\left(N_{2}\right) \otimes S U\left(N_{3}\right) \otimes S U\left(N_{k}\right)$. For this situation we find

$$
\operatorname{dim}_{\mathbb{R}} G=1+\sum_{j=1}^{n}\left(N_{j}^{2}-1\right)=1-n+\sum_{j=1}^{n} N_{j}^{2} .
$$

For generic orbits, the stabilizer subgroups have dimension 0 , so that the real dimension of generic orbits is real $\operatorname{dim} G$. (The dimension of orbits of maximally entangled states is less than real dim $G$ [8].) For $n$ qubits, we have $N_{j}=2$ so the real dimension of the local unitary group that acts on a $n$-qubit vector space is $3 n+1$, allowing for the $U(1)$ factor of overall phase, and this $3 n+1$ is also the dimension of a generic orbit.

The space of orbits of normalized vectors of $\mathcal{H}$ has real dimension equal to $\left(\operatorname{dim}_{\mathbb{R}} \mathcal{H}\right)-1$ less the real dimension of a generic orbit. For $G$ and $\mathcal{H}$ as above

$$
\operatorname{dim}_{\mathbb{R}} \mathcal{H}=2 \prod_{j=1}^{n} N_{j}
$$

and the space of orbits of normalized states, including overall phase, has real dimension

$$
2 \prod_{j=1}^{n} N_{j}-2+n-\sum_{j=1}^{n} N_{j}^{2}
$$

One sees that for large $n$, the orbit dimension is tiny compared to the dimension of the space of orbits.

Note: Reported orbit dimensions can be inconsistent because different papers make different choices concerning whether to include or drop overall phase. Our choice of including the $U(1)$ factor in the local unitary group inflates the real dimension of orbits by 1 compared against other papers that ignore overall phase. The choice of including or dropping overall phase has no effect on the dimension of the stabilizer subgroup. 


\section{Matrix notation for vectors in bipartite tensor-product spaces}

A known matrix notation for bipartite pure states, including those obtained from splittings of multipartite states, proves useful. Consider a finite-dimensional vector space of complex dimension $m n$ thought of as the tensor product space $\mathcal{C}^{m} \otimes \mathcal{C}^{n}$, where $\mathcal{C}^{m}$ denotes the vector space of complex dimension $m$. Any vector in this space has $m n$ components, and these components can be arranged to form an $m$-by- $n$ matrix. Conversely any $m$-by- $n$ matrix $A$ determines a vector in $\mathcal{C}^{m} \otimes \mathcal{C}^{n}$ by the following rule: let $\left.|A\rangle\right\rangle$ be the column vector having $m n$ elements obtained by a raster scan of $A$; that is, one "writes" the elements of the first row of $A$ one under the other, followed by the elements of the second row, and so on [9]:

$$
|A\rangle\rangle=\left[A_{11}, \ldots, A_{1 n}, A_{21}, \ldots, A_{2 n}, A_{31}, \ldots \ldots, A_{m n}\right]^{T},
$$

where the superscript $T$ applied to any rectangular matrix denotes its transpose (without complex conjugation). For any rectangular matrix $A$ let $A^{\dagger}$ be the hermitian conjugate of $A$, and let $\bar{A}$ be its complex conjugate without transpose, so that $A^{\dagger}=\overline{\left(A^{T}\right)}=\bar{A}^{T}$.

Let $M_{m n}$ denote the space of $m$-by- $n$ matrices over complex numbers and abbreviate square matrices $M_{m m}$ by $M_{m}$. Then for $A, B \in M_{m n}, C \in M_{m}$ and $D \in M_{n}$, we have [9]

$$
\begin{aligned}
C \otimes D|A\rangle\rangle & \left.=\left|C A D^{T}\right\rangle\right\rangle, \\
\operatorname{tr}_{2}(|A\rangle\rangle_{1212}\langle\langle B|) & =A B^{\dagger} \in M_{m}, \\
\operatorname{tr}_{1}(|A\rangle\rangle_{1212}\langle\langle B|) & =A^{T} \bar{B} \in M_{n},
\end{aligned}
$$

from which it follows that

$$
\left.\left.\left(I_{m} \otimes A^{T}\right)|C\rangle\right\rangle=\left(C \otimes I_{n}\right)|A\rangle\right\rangle .
$$

In this matrix notation, Eq. (6) becomes

$$
\left.D^{2}(|\psi\rangle\rangle\right)=\langle\langle\psi \mid \psi\rangle\rangle-\Lambda_{\max }\left(\psi \psi^{\dagger}\right)=\langle\langle\psi \mid \psi\rangle\rangle-\Lambda_{\max }\left(\psi^{\dagger} \psi\right),
$$

which implies that

$$
\left.\left.\left.\left.D^{2}(|\psi\rangle\rangle\right)=D^{2}\left(\left|\psi^{\dagger}\right\rangle\right\rangle\right)=D^{2}(|\bar{\psi}\rangle\rangle\right)=D^{2}\left(\left|\psi^{T}\right\rangle\right\rangle\right) .
$$

We also have

$$
\langle\langle\psi \mid \phi\rangle\rangle=\sum_{k=1}^{m} \sum_{\ell=1}^{n} \bar{\psi}_{k \ell} \phi_{k \ell}=\operatorname{tr}\left(\psi^{\dagger} \phi\right)=\operatorname{tr}\left(\phi \psi^{\dagger}\right),
$$

and in particular

$$
\langle\langle\psi \mid \psi\rangle\rangle=\sum_{k=1}^{m} \sum_{\ell=1}^{n}\left|\psi_{k l}\right|^{2}=\operatorname{tr}\left(\psi^{\dagger} \psi\right)=\operatorname{tr}\left(\psi \psi^{\dagger}\right) .
$$

\section{SOME ENTANGLEMENT PROPERTIES OF PURE STATES}

\section{A. Maximum possible degree of pure-state entanglement}

It follows from Eq. (6) that for any unit vector $|\hat{\psi}\rangle\rangle \in \mathcal{H}$ split into two factors $\mathcal{H}_{\mathrm{I}} \otimes \mathcal{H}_{\mathrm{II}}$, the corresponding split degree of entanglement involves the maximum eigenvalue of a reduced density matrix. If $\operatorname{dim}_{\mathbb{C}} \mathcal{H}_{\mathrm{I}} \leq \operatorname{dim}_{\mathbb{C}} \mathcal{H}_{\mathrm{II}}$, the maximally entangled case is that for which

$$
\operatorname{tr}_{\text {II }}(|\hat{\psi}\rangle\rangle\langle\langle\hat{\psi}|)=\psi \psi^{\dagger} \frac{1}{\operatorname{dim}_{\mathbb{C}} \mathcal{H}_{\mathrm{I}}} \mathbf{1}_{\mathcal{H}_{\mathrm{I}}},
$$


where $\psi$ is a $\operatorname{dim}_{\mathbb{C}} \mathcal{H}_{\mathrm{I}}$-by-dim $\mathbb{C} \mathcal{H}_{\mathrm{II}}$ rectangular matrix. In the opposite case of $\operatorname{dim}_{\mathbb{C}} \mathcal{H}_{\mathrm{I}}>$ $\operatorname{dim}_{\mathbb{C}} \mathcal{H}_{\mathrm{II}}$, the reduced density matrix $\psi \psi^{\dagger}$ has the same maximum eigenvalue as $\psi^{\dagger} \psi$ [10], so that in either case the maximum eigenvalue is less than or equal to the inverse of the lesser of $\operatorname{dim}_{\mathbb{C}} \mathcal{H}_{\mathrm{I}}$ and $\operatorname{dim}_{\mathbb{C}} \mathcal{H}_{\mathrm{II}}$. Consequently the square of the degree of entanglement for this split can be no greater than

$$
1-1 / \min \left(\operatorname{dim}_{\mathbb{C}} \mathcal{H}_{\mathrm{I}}, \operatorname{dim}_{\mathbb{C}} \mathcal{H}_{\mathrm{II}}\right)
$$

It is known that this bound is tight. The same logic now extends to show that for $|\hat{\psi}\rangle \in$ $\mathcal{H}=\mathcal{H}_{1} \otimes \mathcal{H}_{2} \otimes \mathcal{H}_{3} \otimes \ldots$, the least split degree of entanglement is tightly bounded above by $1-1 / \min _{j} \operatorname{dim}_{\mathbb{C}} \mathcal{H}_{j}$, whence it follows from the definition of degree of entanglement that the maximum degree of entanglement possible for any such $|\psi\rangle$, denoted $D_{0}(\mathcal{H})$, is given by

$$
D_{0}^{2}\left(\mathcal{H}_{1} \otimes \mathcal{H}_{2}\right)=1-1 / \min _{j} \operatorname{dim}_{\mathbb{C}} \mathcal{H}_{j}
$$

Thus we see:

1. If one or both of $\mathcal{H}_{1}$ and $\mathcal{H}_{2}$ is a single qubit space (with complex dimension 2), the maximum degree of entanglement for a normalized state is $1-1 / 2=1 / 2$.

2. As the dimension of both vector spaces approaches infinity, the maximum possible degree of entanglement approaches 1.

\section{B. Maximally entangled 3-qubit pure states}

We want to find the manifold of all 3-qubit pure states that exhibit the maximal split degree of entanglement for all 3 splittings. This is a subset of the manifold of 3-qubit entangled pure states of the form

$$
|\psi\rangle=\sum_{k=0}^{1} \sum_{\ell=0}^{1} \sum_{m=0}^{1} \psi_{k l m}|k, \ell, m\rangle,
$$

with the normalization

$$
\sum_{k=0}^{1} \sum_{\ell=0}^{1} \sum_{m=0}^{1}\left|\psi_{k l m}\right|^{2}=1
$$

Consider first the splitting $|1\rangle|2,3\rangle$. From Eq. (34) we see that the maximum possible split degree of entanglement is $D_{0}=1 / 2$. For this $1 \mid 23$ splitting, it is convenient to view the 3 -qubit state as $|\psi\rangle\rangle$ where $\psi$ is the 2-by-4 matrix

$$
\psi=\left[\begin{array}{llll}
\psi_{000} & \psi_{001} & \psi_{010} & \psi_{011} \\
\psi_{100} & \psi_{101} & \psi_{110} & \psi_{111}
\end{array}\right]
$$

Then from Eq. (28) the square of the degree of entanglement is

$$
D_{1 \mid 23}^{2}=1-\Lambda_{\max }\left[\operatorname{tr}_{2}(|\psi\rangle\rangle\langle\langle\psi|)\right]=1-\Lambda_{\max }\left(\psi \psi^{\dagger}\right)
$$

and from Eq. (32) the maximal degree of entanglement $D_{1 \mid 23}=1 / 2$ is achieved if and only if

$$
\psi \psi^{\dagger}=\frac{1}{2} \mathbf{1}_{2}=\frac{1}{2}\left[\begin{array}{ll}
1 & 0 \\
0 & 1
\end{array}\right] .
$$


By explicitly writing out $\psi \psi^{\dagger}$ and comparing the result with Eq. (39) one obtains the four equations

$$
\begin{aligned}
& \left(\psi \psi^{\dagger}\right)_{00}=\left|\psi_{000}\right|^{2}+\left|\psi_{001}\right|^{2}+\left|\psi_{010}\right|^{2}+\left|\psi_{011}\right|^{2}=1 / 2, \\
& \left(\psi \psi^{\dagger}\right)_{01}=\psi_{000} \bar{\psi}_{100}+\psi_{001} \bar{\psi}_{101}+\psi_{010} \bar{\psi}_{110}+\psi_{011} \bar{\psi}_{111}=0 \text {, } \\
& \left(\psi \psi^{\dagger}\right)_{10}=\overline{\left(\psi \psi^{\dagger}\right)_{01}}, \\
& \left(\psi \psi^{\dagger}\right)_{11}=\left|\psi_{100}\right|^{2}+\left|\psi_{101}\right|^{2}+\left|\psi_{110}\right|^{2}+\left|\psi_{111}\right|^{2}=1 / 2 \text {. }
\end{aligned}
$$

The other two splittings give equations with cyclic permutations of the three indices.

To show that all these equations indeed have solutions, we study the special case of symmetry under permutation of the 3 qubits, so that a state is defined by complex parameters $A_{0}, \ldots, A_{3}$ with

$$
A_{0}=\psi_{000}, A_{1}=\psi_{100}=\psi_{010}=\psi_{001}, A_{2}=\psi_{011}=\psi_{101}=\psi_{110}, A_{3}=\psi_{111} .
$$

Equations (40) become

$$
\begin{aligned}
\left|A_{0}\right|^{2}+2\left|A_{1}\right|^{2}+\left|A_{2}\right|^{2} & =1 / 2, \\
\left|A_{1}\right|^{2}+2\left|A_{2}\right|^{2}+\left|A_{3}\right|^{2} & =1 / 2, \\
A_{0} \bar{A}_{1}+2 A_{1} \bar{A}_{2}+A_{2} \bar{A}_{3} & =0 .
\end{aligned}
$$

The local boson-symmetry preserving subgroup of $L U_{3}$ consists of matrices that "act the same" on each factor. That is, the subgroup, denoted $\operatorname{Bos} L U_{3}$, with elements of the form

$$
e^{i \phi}\left[\begin{array}{cc}
\alpha & \beta \\
-\bar{\beta} & \bar{\alpha}
\end{array}\right]^{\otimes 3}
$$

with $|\alpha|^{2}+|\beta|^{2}=1$. A direct calculation shows that, regardless of the degree of entanglement, any 3-qubit pure bosonic state is in an orbit under Bos $L U_{3}$ containing a representative state (hence having the same degree of entanglement) for which

$$
\left|A_{0}\right|=\left|A_{3}\right| \text {. }
$$

Adjoining Eq. (46) to Eqs. (42)-(44), we solve for the representative state of the general orbit of a maximally entangled state, and discover immediately that for this representative state $\left|A_{1}\right|=\left|A_{2}\right|$. The problem of determining the representative then splits into two cases.

Case 1: $A_{1}=A_{2}=0$ : For this case, we invoke freedom under local unitary transforms of the form $e^{i \phi}\left[\begin{array}{cc}e^{i \theta} & 0 \\ 0 & e^{-i \theta}\end{array}\right]^{\otimes 3}$ to make $A_{0}$ and $A_{3}$ real and non-negative, resulting in the GHZ state $|\mathrm{GHZ}\rangle=2^{-1 / 2}[1,0,0,0,0,0,0,1]^{T}$.

Case 2: $\left|A_{1}\right|=\left|A_{2}\right| \neq 0$ : For this case, the phase freedom allows one to make $A_{1}$ and $A_{2}$ real and positive, so that for some real, positive $x$ we have $A_{1}=A_{2}=x$. Accompanied by this additional condition, Eq. (44) implies

$$
A_{0}+2 x+\bar{A}_{3}=0
$$

implying that $\operatorname{Im} A_{0}=\operatorname{Im} A_{3}$, which in turn forces the conclusion that $\operatorname{Re} A_{0}=\operatorname{Re} A_{3}=-x$. Thus we find

$$
|\psi\rangle=[-x+i y, x, x, x, x, x, x,-x+i y]^{T},
$$

where the real parameters $x$ and $y$ satisfy $0 \leq x \leq 1 / \sqrt{8}$ and $y= \pm \sqrt{\frac{1}{2}-4 x^{2}}$. Direct calculation using Bos $L U_{3}$ shows that Cases 1 and 2 give representatives of the same orbit, so the set of 3-qubit maximally entangled states with bosonic symmetry is the orbit under Bos $L U_{3}$ of the GHZ state. This orbit has real dimension 4 and consists of states of the form 


$$
|\psi\rangle=2^{-1 / 2} e^{i \phi}\left[\begin{array}{cc}
\alpha & \beta \\
-\bar{\beta} & \bar{\alpha}
\end{array}\right]^{\otimes 3}\left[\begin{array}{l}
1 \\
0 \\
0 \\
0 \\
0 \\
0 \\
0 \\
1
\end{array}\right]=2^{-1 / 2} e^{i \phi}\left[\begin{array}{c}
\alpha^{3}+\beta^{3} \\
-\alpha^{2} \bar{\beta}+\bar{\alpha} \beta^{2} \\
-\alpha^{2} \bar{\beta}+\bar{\alpha} \beta^{2} \\
\alpha \bar{\beta}^{2}+\bar{\alpha}^{2} \beta \\
-\alpha^{2} \bar{\beta}+\bar{\alpha} \beta^{2} \\
\alpha \bar{\beta}^{2}+\bar{\alpha}^{2} \beta \\
\alpha \bar{\beta}^{2}+\bar{\alpha}^{2} \beta \\
\bar{\alpha}^{3}-\bar{\beta}^{3}
\end{array}\right]
$$

where $|\alpha|^{2}+|\beta|^{2}=1$.

Without the constraint of bosonic symmetry, the orbit of the GHZ state under $U(1) \otimes S U(2)^{\otimes 3}$ consists of states of the form

$$
|\psi\rangle=e^{i \phi}\left[\begin{array}{cc}
\alpha_{1} & \beta_{1} \\
-\bar{\beta}_{1} & \bar{\alpha}_{1}
\end{array}\right] \otimes\left[\begin{array}{cc}
\alpha_{2} & \beta_{2} \\
-\bar{\beta}_{2} & \bar{\alpha}_{2}
\end{array}\right] \otimes\left[\begin{array}{cc}
\alpha_{3} & \beta_{3} \\
-\bar{\beta}_{3} & \bar{\alpha}_{3}
\end{array}\right]|\mathrm{GHZ}\rangle,
$$

where $\left|\alpha_{i}\right|^{2}+\left|\beta_{i}\right|^{2}=1, i=1,2,3$. Because the stabilizer subgroup for the GHZ state has 2 real parameters, the orbit has real dimension 8, in agreement with the known manifold of 3-qubit maximally entangled states [8].

\section{Pure 4-qubit symmetric states}

We analyzed four-qubit pure states symmetric under permutation of qubits, which have the form

$$
|\psi\rangle=\left[A_{0}, A_{1}, A_{1}, A_{2}, A_{1}, A_{2}, A_{2}, A_{3}, A_{1}, A_{2}, A_{2}, A_{3}, A_{2}, A_{3}, A_{3}, A_{4}\right]^{T} .
$$

Because of this permutation symmetry we have for all the " $1-3$ " splittings between a one-qubit factor and the other three qubits taken together

$$
D_{1 \mid 234}=D_{2 \mid 134}=D_{3 \mid 124}=D_{4 \mid 123} .
$$

Since the smaller space in the 1-3 split is a single qubit, the maximum possible value of $D_{1 \mid 234}^{2}$ is $1 / 2$, as shown above, and this value is attained, following the procedure of Sec. IV, when $\operatorname{tr}_{234}(|\psi\rangle\langle\psi|)=\frac{1}{2} \mathbf{1}_{2}$, which is the case if and only if

$$
\begin{aligned}
\left|A_{0}\right|^{2}+3\left|A_{1}\right|^{2}+3\left|A_{2}\right|^{2}+\left|A_{3}\right|^{2} & =1 / 2, \\
\left|A_{1}\right|^{2}+3\left|A_{2}\right|^{2}+3\left|A_{3}\right|^{2}+\left|A_{4}\right|^{2} & =1 / 2, \\
A_{0} \bar{A}_{1}+3 A_{1} \bar{A}_{2}+3 A_{2} \bar{A}_{3}+A_{3} \bar{A}_{4} & =0 .
\end{aligned}
$$

Without regard to the "2-2" splitting between two qubits and the other two qubits, which by symmetry all have the same degree of entanglement $D_{12 \mid 34}$, we explicitly solve Eqs. (53)-(55) for the manifold of all states of maximal $D_{1 \mid 234}=1 / \sqrt{2}$ as follows. A direct calculation shows that any 4-qubit bosonic pure state lies in an orbit under Bos $L U_{4}$ containing a representative state for which

$$
\left|A_{0}\right|=\left|A_{4}\right|
$$


Adjoining Eq. (56) to Eqs. (53)-(55) forces the relation

$$
\left|A_{1}\right|=\left|A_{3}\right|
$$

which leads to two cases with the following solutions. For the $A_{1}=A_{2}=0$ case, we find

$$
A_{0}=A_{4}= \pm \sqrt{\frac{1}{2}-3\left|A_{2}\right|^{2}}
$$

For the $A_{1}=A_{2} \neq 0$ case, phase freedom allows one to make $A_{1}=A_{3}=x_{1} \geq 0$, and we then get two solutions. The first solution is

$$
A_{0}=x_{0}+i y_{0}, \quad A_{1}=A_{3}=x_{1}, \quad A_{2}=-i y_{2}, \quad A_{4}=-\bar{A}_{0},
$$

subject to the constraint that

$$
x_{0}^{2}+y_{0}^{2}+4 x_{1}^{2}+3 y_{2}^{2}=1 / 2
$$

the second solution is

$$
A_{0}=A_{4}=-3 x_{2}+i y_{0}, \quad A_{1}=A_{3}=x_{1}, \quad A_{2}=x_{2}+i y_{2},
$$

subject to the constraint that

$$
y_{0}^{2}+4 x_{1}^{2}+12 x_{2}^{2}+3 y_{2}^{2}=1 / 2 .
$$

Both the first and second solutions involve $4-1=3$ real parameters. We thus see distinct types of maximally symmetric 4-qubit states, and in Sec. VI.2 we discuss varying degrees of 2-2 entanglement that can be exhibited by maximally 1-3 entangled states, with implications for reduced density matrices obtained from symmetric pure 4-qubit states.

\section{CALCULATING DEGREES OF ENTANGLEMENT OF MIXED STATES}

\section{A. Minimizing decompositions of a mixed state}

In this subsection a variational procedure demonstrates two key propositions pertaining to minimizing decompositions that define the degree of entanglement of mixed states, as in Eq. (10). Consider a pair of terms involving the unnormalized states of a decomposition for any particular splitting, as in Eq. (9). If such a decomposition is expressed in rectangular matrices $\psi_{j}$, we have

$$
\left.\rho=\sum_{j}\left|\psi_{j}\right\rangle\right\rangle\left\langle\left\langle\psi_{j}\right| .\right.
$$

If this decomposition minimizes the weighted sum of degrees of entanglement of its terms, it also minimizes the weighted sum taken over any pair of terms, which we now study in detail. If the sum of any two selected terms is denoted by $\chi$, there are rectangular matrices $x$ and $y$ such that

$$
\chi=|x\rangle\rangle\langle\langle x|+| y\rangle\rangle\langle\langle y| \text {. }
$$

Important is the fact that if another pair $\tilde{x}$ and $\tilde{y}$ combine to make the same $\chi$, the sum of the degrees of entanglement may differ:

$$
\left.\left.|x\rangle\rangle\langle\langle x|+| y\rangle\rangle\langle\langle y|=| \tilde{x}\rangle\rangle\langle\langle\tilde{x}|+| \tilde{y}\rangle\rangle\left\langle\left\langle\tilde{y} \mid \not \Rightarrow D^{2}(|x\rangle\rangle\right)+D^{2}(|y\rangle\rangle\right)=D^{2}(|\tilde{x}\rangle\rangle\right)+D^{2}(|\tilde{y}\rangle\rangle\right) .
$$


For the sum $\left.\left.D^{2}(|x\rangle\rangle\right)+D^{2}(|y\rangle\rangle\right)$ to be a minimum under the constraint that $\chi$ be invariant, the first variation of $\chi=|x\rangle\rangle\langle\langle x|+| y\rangle\rangle\langle\langle y|$, subject to the same constraint, must vanish. To calculate the desired variation, we make use of the invariance of $\chi$ under a unitary transformation to $|x\rangle\rangle$ and $|y\rangle\rangle$; that is, for any 2-by-2 unitary matrix $W$ we define

$$
\left[\begin{array}{l}
\tilde{x} \\
\tilde{y}
\end{array}\right]=W\left[\begin{array}{l}
x \\
y
\end{array}\right] .
$$

The transformation satisfies the needed constraint; i.e.,

$$
\chi=|x\rangle\rangle\langle\langle x|+| y\rangle\rangle\langle\langle y|=| \tilde{x}\rangle\rangle\langle\langle\tilde{x}|+| \tilde{y}\rangle\rangle\langle\langle\tilde{y}| .
$$

Multiplying $W$ on the left by $\operatorname{diag}\left(e^{i \alpha}, e^{i \beta}\right)$ leaves the degree of entanglement of $\left.|\tilde{x}\rangle\right\rangle$ and $\left.|\tilde{y}\rangle\right\rangle$ unchanged, so the relevant infinitesimal $U(2)$ transformation has the form

$$
W=\left[\begin{array}{cc}
1 & \theta \\
-\bar{\theta} & 1
\end{array}\right]
$$

and we find

$$
\tilde{x}=x+\theta y, \quad \tilde{y}=y-\bar{\theta} x
$$

Equation (28) implies

$$
\begin{aligned}
\left.\left.D^{2}(|\tilde{x}\rangle\rangle\right)+D^{2}(|\tilde{y}\rangle\rangle\right) & =\langle\langle\tilde{x} \mid \tilde{x}\rangle\rangle+\langle\langle\tilde{y} \mid \tilde{y}\rangle\rangle-\Lambda_{\max }\left(\tilde{x} \tilde{x}^{\dagger}\right)-\Lambda_{\max }\left(\tilde{y} \tilde{y}^{\dagger}\right) \\
& =\langle\langle x \mid x\rangle\rangle+\langle\langle y \mid y\rangle\rangle-\Lambda_{\max }\left(\tilde{x} \tilde{x}^{\dagger}\right)-\Lambda_{\max }\left(\tilde{y} \tilde{y}^{\dagger}\right) .
\end{aligned}
$$

For the minimizing decomposition, the first variation of $\left.\left.D^{2}(|\tilde{x}\rangle\rangle\right)+D^{2}(|\tilde{y}\rangle\rangle\right)$ with respect to $\theta$ must vanish. Consider

$$
\begin{aligned}
\Lambda_{\max }\left(\tilde{x} \tilde{x}^{\dagger}\right) & =\Lambda_{\max }\left[(x+\theta y)(x+\bar{\theta} y)^{\dagger}\right] \\
& =\Lambda_{\max }\left[x x^{\dagger}+\theta y x^{\dagger}+\bar{\theta} x y^{\dagger}+\mathrm{O}(\theta \bar{\theta})\right] .
\end{aligned}
$$

To find the variation of the eigenvalues we apply first-order perturbation theory [11]. Assume that the maximum eigenvalue of $x x^{\dagger}$ has a 1-dimensional eigenspace (if not, shift $x$ to a nearby generic matrix that has this property). Let $\left|\lambda_{x}\right\rangle$ be the normalized eigenvector for the maximum eigenvalue (denoted $\lambda_{x}$ ) of $x x^{\dagger}$, so that

$$
x x^{\dagger}\left|\lambda_{x}\right\rangle=\lambda_{x}\left|\lambda_{x}\right\rangle
$$

From first-order perturbation theory we have, to within terms of second order,

$$
\Lambda_{\max }\left(\tilde{x} \tilde{x}^{\dagger}\right)=\Lambda_{\max }\left(x x^{\dagger}\right)+\left\langle\lambda_{x}\left|\left(\theta y x^{\dagger}+\bar{\theta} x y^{\dagger}\right)\right| \lambda_{x}\right\rangle .
$$

Similarly one finds (to within terms of second order in $|\theta|$ )

$$
\Lambda_{\max }\left(\tilde{y} \tilde{y}^{\dagger}\right)=\Lambda_{\max }\left(y y^{\dagger}\right)-\left\langle\lambda_{y}\left|\left(\theta y x^{\dagger}+\bar{\theta} x y^{\dagger}\right)\right| \lambda_{y}\right\rangle .
$$

Treating the variations in $\theta$ and $\bar{\theta}$ as independent yields the following

Proposition: For any density matrix $\rho$ and any split degree of entanglement of $\rho$, a minimizing decomposition that includes the two terms $|x\rangle\rangle$ and $|y\rangle\rangle$ is constrained to satisfy

$$
\left\langle\lambda_{x}\left|y x^{\dagger}\right| \lambda_{x}\right\rangle-\left\langle\lambda_{y}\left|y x^{\dagger}\right| \lambda_{y}\right\rangle=0
$$


Equivalently one has the two relations obtained by splitting $x x^{\dagger}$ into hermitian and anti-hermitian terms:

$$
\begin{aligned}
\left\langle\lambda_{j}\left|\left(x_{j} x_{k}^{\dagger}+x_{k} x_{j}^{\dagger}\right)\right| \lambda_{j}\right\rangle & =\left\langle\lambda_{k}\left|\left(x_{j} x_{k}^{\dagger}+x_{k} x_{j}^{\dagger}\right)\right| \lambda_{k}\right\rangle, \\
\left\langle\lambda_{j}\left|i\left(x_{j} x_{k}^{\dagger}-x_{k} x_{j}^{\dagger}\right)\right| \lambda_{j}\right\rangle & =\left\langle\lambda_{k}\left|i\left(x_{j} x_{k}^{\dagger}-x_{k} x_{j}^{\dagger}\right)\right| \lambda_{k}\right\rangle .
\end{aligned}
$$

(The matrices $x_{j} x_{k}^{\dagger}$ are all $\operatorname{dim}_{\mathbb{C}} \mathcal{H}_{1}$ by $\operatorname{dim}_{\mathbb{C}} \mathcal{H}_{1}$.)

\section{B. Implications for degrees of entanglement of a given density matrix}

We consider the split degree of entanglement between the first factor and the rest of the factors of $\mathcal{H}$. Any density matrix $\rho$ on a tensor product space split into pieces $\mathcal{H}=\mathcal{H}_{\mathrm{I}} \otimes \mathcal{H}_{\mathrm{II}}$ has a decomposition, not necessarily minimal, of the form

$$
\left.\rho=\sum_{j=1}^{\operatorname{rank} \rho}\left|\psi_{j}\right\rangle\right\rangle\left\langle\left\langle\psi_{j}\right|,\right.
$$

where the matrices $\psi_{j}$ are $\operatorname{dim}_{\mathbb{C}} \mathcal{H}_{\mathrm{I}}$ by $\operatorname{dim}_{\mathbb{C}} \mathcal{H}_{\mathrm{II}}$. Denote the corresponding split degree of entanglement by $D_{\text {split }}(\rho)$, defined in terms of a minimizing decomposition where the minimum ranges over the number $L$ of terms as well as the terms themselves so that we have

$$
\left.D_{\text {split }}^{2}(\rho)=\sum_{j=1}^{L} D_{\text {split }}^{2}\left(\left|x_{j}\right\rangle\right\rangle\right),
$$

under the constraint that

$$
\left.\sum_{j=1}^{L}\left|x_{j}\right\rangle\right\rangle\left\langle\left\langle x_{j}\right|=\rho\right.
$$

From [12], it follows that

$$
x_{j}=\sum_{k=1}^{\operatorname{rank} \rho} w_{j k}^{\prime} \psi_{k} \quad \text { for } j=1, \ldots, L,
$$

for some coefficients $w_{j k}^{\prime}$ that satisfy the orthogonality relations

$$
\sum_{j=1}^{L} w_{j k}^{\prime} \bar{w}_{j \ell}^{\prime}=\delta_{k \ell}
$$

Notice that Eq. (79) is invariant under the phase change

$$
w_{j k}^{\prime} \rightarrow w_{j k}=e^{i \phi_{j}} w_{j k}^{\prime} .
$$

Now we count parameters. Let $R=\operatorname{rank} \rho$. There are $L R$ complex coefficients $w_{j k}^{\prime}$ and so $2 L R$ real coefficients less $R(R-1)$ real orthogonality relations between rows of the $w$ matrix less $R$ normalization conditions on columns less $L$ phase invariances for rows (per Eq. (82)) producing

$$
\text { number of free real parameters }=2 R L-R^{2}-L \text {. }
$$


From Eq. (76) we get $2 \times L(L-1) / 2=L(L-1)$ real relations that constrain the $w_{j k}^{\prime}$. If the number of parameters is greater than the number of equations, we are free to set some of the $x_{j}$ to zero and compensate by multiplying others by a constant. Thus we arrive at the following

Proposition: There is always a minimizing decomposition for $D_{\text {split }}(\rho)$ with

$$
L=\operatorname{rank} \rho .
$$

\section{Case of density matrix $\rho$ of rank 2}

To proceed consider the case in which $\rho$ has rank 2, and hence $L=2$. Using the phase freedom expressed in Eq. (82) without loss of generality we take for the most general decomposition

$$
\text { (for } j=1, \ldots, L) \quad w_{j 1}^{\prime}=e^{-\left(i \gamma_{j} / 2\right)} w_{j 1} \quad \text { and } \quad w_{j 2}^{\prime}=e^{\left(i \gamma_{j} / 2\right)} w_{j 2} \text {, }
$$

with $w_{j 1}$ and $w_{j_{2}}$ real and non-negative. For this case of rank 2, the constraint of Eq. (81) and the phase freedom of Eq. (85) give

$$
\left[\begin{array}{ll}
w_{11}^{\prime} & w_{12}^{\prime} \\
w_{21}^{\prime} & w_{22}^{\prime}
\end{array}\right]=\left[\begin{array}{rr}
e^{i \gamma / 2} \cos \left(\frac{1}{2} \theta\right) & e^{-i \gamma / 2} \sin \left(\frac{1}{2} \theta\right) \\
-e^{i \gamma / 2} \sin \left(\frac{1}{2} \theta\right) & e^{-i \gamma / 2} \cos \left(\frac{1}{2} \theta\right)
\end{array}\right],
$$

where $\theta$ and $\gamma$ are parameters. From Eq. (80), it then follows that

$$
\begin{aligned}
& x_{1}=e^{i \gamma / 2} \cos \left(\frac{1}{2} \theta\right) \psi_{1}+e^{-i \gamma / 2} \sin \left(\frac{1}{2} \theta\right) \psi_{2}, \\
& x_{2}=-e^{i \gamma / 2} \sin \left(\frac{1}{2} \theta\right) \psi_{1}+e^{-i \gamma / 2} \cos \left(\frac{1}{2} \theta\right) \psi_{2} .
\end{aligned}
$$

Especially for the case of rank 2, it is convenient to put Eq. (75) in the form

$$
0=\operatorname{tr}\left[x_{1} x_{2}^{\dagger}\left(\left|\lambda_{1}\right\rangle\left\langle\lambda_{1}|-| \lambda_{2}\right\rangle\left\langle\lambda_{2}\right|\right)\right] .
$$

One needs the eigenvectors for $\left|\lambda_{1}\right\rangle$ and $\left|\lambda_{2}\right\rangle$ for $x_{1} x_{1}^{\dagger}$ and $x_{2} x_{2}^{\dagger}$, respectively. Any 2-by-2 hermitian matrix $M$ has eigenvalues $\mu_{1} \geq \mu_{2}$, with corresponding normalized eigenvectors $\left|\mu_{1}\right\rangle$ and $\left|\mu_{2}\right\rangle$ so that $M=\mu_{1}\left|\mu_{1}\right\rangle\left\langle\mu_{1}\left|+\mu_{2}\right| \mu_{2}\right\rangle\left\langle\mu_{2}\right|$. Because $\left|\mu_{1}\right\rangle\left\langle\mu_{1}|+| \mu_{2}\right\rangle\left\langle\mu_{2}\right|=1$, we obtain with a little algebra for distinct eigenvalues

$$
\left|\mu_{1}\right\rangle\left\langle\mu_{1}\right|=\frac{M-\mu_{2} \mathbf{1}}{\mu_{1}-\mu_{2}},
$$

whence it follows that

$$
\left|\lambda_{1}\right\rangle\left\langle\lambda_{1}|-| \lambda_{2}\right\rangle\left\langle\lambda_{2}\right|=\frac{x_{1} x_{1}^{\dagger}-\frac{1}{2} \operatorname{tr}\left(x_{1} x_{1}^{\dagger}\right)}{\sqrt{\operatorname{tr}^{2}\left(x_{1} x_{1}^{\dagger}\right)-4 \operatorname{det}\left(x_{1} x_{1}^{\dagger}\right)}}-\frac{x_{2} x_{2}^{\dagger}-\frac{1}{2} \operatorname{tr}\left(x_{2} x_{2}^{\dagger}\right)}{\sqrt{\operatorname{tr}^{2}\left(x_{2} x_{2}^{\dagger}\right)-4 \operatorname{det}\left(x_{2} x_{2}^{\dagger}\right)}} .
$$

With $x_{j}=x_{j}(\theta, \gamma)$ as given in Eqs. (87), one then determines the values of $\theta$ and $\gamma$ by solving the complex-valued equation

$$
0=\operatorname{tr}\left[x_{1} x_{2}^{\dagger}\left(\frac{x_{1} x_{1}^{\dagger}-\frac{1}{2} \operatorname{tr}\left(x_{1} x_{1}^{\dagger}\right)}{\sqrt{\operatorname{tr}^{2}\left(x_{1} x_{1}^{\dagger}\right)-4 \operatorname{det}\left(x_{1} x_{1}^{\dagger}\right)}}-\frac{x_{2} x_{2}^{\dagger}-\frac{1}{2} \operatorname{tr}\left(x_{2} x_{2}^{\dagger}\right)}{\sqrt{\operatorname{tr}^{2}\left(x_{2} x_{2}^{\dagger}\right)-4 \operatorname{det}\left(x_{2} x_{2}^{\dagger}\right)}}\right)\right] .
$$

Plugging the values of $\theta$ and $\gamma$ that solve this equation (in a numerical analysis) back into Eqs. (87) determines $x_{1}$ and $x_{2}$ such that for our case of rank 2, we have per Eq. (78)

$$
\left.\left.D_{\text {split }}^{2}(\rho)=D_{\text {split }}^{2}\left(\left|x_{1}\right\rangle\right\rangle\right)+D_{\text {split }}^{2}\left(\left|x_{2}\right\rangle\right\rangle\right) .
$$

We checked that numerical solutions for the case of 2 qubits agree with the result obtained from Eq. (12). 


\section{SURVIVAL OF ENTANGLEMENT UNDER PARTIAL TRACE}

The partial trace over one factor of a normalized multipartite pure state yields a reduced density matrix. For a general multipartite pure state $|\psi\rangle$ and the density matrix $\rho$ obtained as one or another partial trace of $|\psi\rangle\langle\psi|$, in some cases one finds $D(\rho)$ less than $D(|\psi\rangle)$ while in other cases $D(\rho)>D(|\psi\rangle)$. An example of the latter situation is the 3-qubit state $\left|\psi_{3}\right\rangle$ consisting of the tensor product of a single qubit state with a 2-qubit Bell state. Being a product state, this $\left|\psi_{3}\right\rangle$ has zero degree of entanglement while the 2-qubit $\rho_{2}$ obtained by tracing over the single qubit has the maximum possible degree of entanglement.

A systematic relation is imposed by bosonic symmetry. For all bosonic states pure or mixed, expressed by a density matrix $\rho$, we have

$$
D\left(\operatorname{tr}_{1} \rho\right) \leq D(\rho) .
$$

Proof: Any density matrix $\rho$ has a minimizing decomposition $\left\{\left|\psi_{j}\right\rangle\right\}$, as in Eq. (10), such that $\rho=\sum_{j}\left|\psi_{j}\right\rangle\left\langle\psi_{j}\right|$ and $D^{2}(\rho)=\sum_{j} D^{2}\left(\left|\psi_{j}\right\rangle\right)$. Then we have

$$
\operatorname{tr}_{1} \rho=\sum_{j} \operatorname{tr}_{1}\left(\left|\psi_{j}\right\rangle\left\langle\psi_{j}\right|\right)
$$

Equation (15) implies that

$$
D^{2}\left(\operatorname{tr}_{1} \rho\right) \leq \sum_{j} D^{2}\left[\operatorname{tr}_{1}\left(\left|\psi_{j}\right\rangle\left\langle\psi_{j}\right|\right)\right] .
$$

As shown in Appendix B.3, for any pure-state $\left|\psi_{j}\right\rangle$ having bosonic symmetry

$$
D\left(\operatorname{tr}_{1}\left(\left|\psi_{j}\right\rangle\left\langle\psi_{j}\right|\right)\right) \leq D\left(\left|\psi_{j}\right\rangle\right),
$$

whence we have

$$
D^{2}\left(\operatorname{tr}_{1} \rho\right) \leq \sum_{j} D^{2}\left(\left|\psi_{j}\right\rangle\right)=D^{2}(\rho)
$$

as desired.

\section{A. Degree of entanglement of partial trace of a bosonic 3-qubit state}

For a maximally entangled symmetric 3-qubit pure state, we study the degree of entanglement of the two-qubit density matrix obtained from a partial trace over one qubit, say the first one. This density operator is obtained as partial trace $\operatorname{tr}_{1}(|\psi\rangle\langle\psi|)$ over a 3-qubit pure state $|\psi\rangle$. Because the partial trace is over a single qubit which is the smaller of the two factor spaces involved in the $1 \mid 23$ splitting, the remark above does not apply, so our first question is how much entanglement survives the partial trace in case the 3-qubit state $|\psi\rangle$ has the maximal possible degree of entanglement of $1 / 2$ ? The answer is provided by a direct calculation of the concurrence of the reduced state

$\rho \stackrel{\text { def }}{=} \operatorname{tr}_{1}(|\psi\rangle\langle\psi|)$. The condition that $|\psi\rangle$ be symmetric under permutations of qubits imposes on the 2-qubit reduced density matrix the condition that the second column equal the third column and that the second row equal the third row:

$$
\rho_{2 j}=\rho_{3 j} \quad \text { and } \quad \rho_{j 2}=\rho_{j 3}, \quad \text { for } \quad j=1, \ldots, 4 .
$$


The condition of being maximally entangled implies Eqs. (42)-(44) which in turn imply additional constraints:

$$
\rho_{24}=-\rho_{12} \quad \text { and } \quad \rho_{11}+\rho_{22}=\rho_{33}+\rho_{44}=1 / 2,
$$

which with Eq. (98) and the hermiticity of density operators impose the form on $\rho$ reduced from a symmetric, maximally entangled 4-qubit state:

$$
\rho=\left[\begin{array}{rrrr}
\frac{1}{2}-\rho_{22} & \rho_{12} & \rho_{12} & \rho_{14} \\
\bar{\rho}_{12} & \rho_{22} & \rho_{22} & -\rho_{12} \\
\bar{\rho}_{12} & \rho_{22} & \rho_{22} & -\rho_{12} \\
\bar{\rho}_{14} & -\bar{\rho}_{12} & -\bar{\rho}_{12} & \frac{1}{2}-\rho_{22}
\end{array}\right] .
$$

We calculate the degree of entanglement of this 2-qubit density operator by calculating its concurrence [7], which involves the square roots of eigenvalues of the operator $R \stackrel{\text { def }}{=} \rho\left(\sigma_{2} \otimes \sigma_{2}\right) \bar{\rho}\left(\sigma_{2} \otimes \sigma_{2}\right)$, where the Pauli matrices are defined by

$$
\sigma_{1}=\left[\begin{array}{ll}
0 & 1 \\
1 & 0
\end{array}\right], \quad \sigma_{2}=\left[\begin{array}{rr}
0 & -i \\
i & 0
\end{array}\right], \quad \sigma_{3}=\left[\begin{array}{rr}
1 & 0 \\
0 & -1
\end{array}\right] .
$$

Direct calculation shows the startling result for a density operator of the form of Eq. (100) that

$$
\left(\sigma_{2} \otimes \sigma_{2}\right) \bar{\rho}\left(\sigma_{2} \otimes \sigma_{2}\right)=\rho .
$$

Thus, the square roots of the eigenvalues of $R$ are just the eigenvalues of $\rho$. Under these strong symmetry conditions on $\rho$ one has for its concurrence $C(\rho)$ :

$$
C(\rho)=\max \left\{2 \Lambda_{\max }(\rho)-\operatorname{tr} \rho, 0\right\}=\max \left\{2 \Lambda_{\max }(\rho)-1,0\right\} .
$$

$D(\rho)$ then follows from Eq. (12). But from Eq. (32) it follows that $\Lambda_{\max }(\rho)=1 / 2$, which via Eq. (103) implies $C(\rho)=0$. From this via Eq. (12) it follows that the density matrix obtained from a partial trace of a single qubit of a maximally entangled symmetric 3-qubit pure state has zero degree of entanglement. Because all the maximally entangled 3-qubit pure states lie in a single orbit under local unitary transformations, we have the stronger statement that for no maximally entangled 3-qubit pure state does any entanglement survive the partial trace over any one qubit.

How about if the 3-qubit symmetric pure state is less than maximally entangled? We wanted to see what symmetric multipartite pure states best survived a partial trace over one factor. To this end we explored the partial traces over symmetric 3-qubit states to determine the degree of entanglement of the reduced density matrices. A general normalized bosonic 3-qubit state has the form:

$$
\begin{aligned}
|\psi\rangle & =\left[\psi_{000}, \psi_{001}, \psi_{010}, \psi_{011}, \psi_{100}, \psi_{101}, \psi_{110}, \psi_{111}\right]^{T} \\
& =\left[A_{0}, A_{1}, A_{1}, A_{2}, A_{1}, A_{2}, A_{2}, A_{3}\right]^{T} .
\end{aligned}
$$

For any bosonic 3-qubit $|\psi\rangle$ there is always some choice of $\gamma$ and $\phi$ such that the local bosonic transformation

$$
\left[\begin{array}{cc}
e^{i \gamma / 2} \cos \left(\frac{1}{2} \theta\right) & e^{-i \gamma / 2} \sin \left(\frac{1}{2} \theta\right) \\
-e^{i \gamma / 2} \sin \left(\frac{1}{2} \theta\right) & e^{-i \gamma / 2} \cos \left(\frac{1}{2} \theta\right)
\end{array}\right]^{\otimes 3}
$$

nulls out $A_{2}$. Further simplification comes because, without disturbing the nulled-out elements, there is always a diagonal local bosonic transformation that puts the state in the form

$$
|\psi\rangle\rangle=\left[x_{0}, x_{1}, x_{1}, 0, x_{1}, 0,0, A_{3}\right]^{T},
$$


where

$$
\psi \stackrel{\text { def }}{=}\left[\begin{array}{cccc}
x_{0} & x_{1} & x_{1} & 0 \\
x_{1} & 0 & 0 & A_{3}
\end{array}\right]
$$

with real-valued $x_{0}, x_{1} \geq 0$ and a normalization condition is imposed so that $x_{0}^{2}+3 x_{1}^{2}+\left|A_{3}\right|^{2}=1$. The real dimension of the orbit space of normalized bosonic pure 3 -qubit states is $8-1-4=3$. Correspondingly our family of representatives has real dimension 4 less a normalization condition and so also has 3 real parameters. No family of representatives of orbits can have fewer parameters.

A 2-qubit reduced density matrix obtained from partial traces of an $n$-qubit bosonic pure state always has two rows identical and two columns identical, and thus has rank of at most 3 . As a result such a 4-by-4 matrix has an eigenvector with 1 in the place acted on by one such column, -1 in the other such place, and two 0 -elements. This eigenvector then has eigenvalue 0 . Leaving this eigenvector out allows one to reduce the $4 \times 4$ matrix to $3 \times 3$.

Using this reduction we explicitly calculated the degree of entanglement of the 2-qubit reduced density matrix obtained by a partial trace over one qubit of the 3-qubit state $|\psi\rangle$ of the form of Eq. (105). Let

$$
a \stackrel{\text { def }}{=}-2 x_{1}^{2}, \quad b \stackrel{\text { def }}{=} 2 x_{1} A_{3}, \quad \text { and } \quad c \stackrel{\text { def }}{=} x_{0} A_{3} .
$$

Then the concurrence of the reduced density matrix defined in [7] is

$$
C^{2}(\rho)=|a|^{2}+|b|^{2}+2|c|^{2}-2\left|a b-c^{2}\right|
$$

and we obtain the degree of entanglement of the 2-qubit mixed state $\rho \stackrel{\text { def }}{=} \operatorname{tr}_{1}(|\psi\rangle\rangle\langle\langle\psi|)$ by

$$
D^{2}(\rho)=\frac{1}{2}\left(1-\sqrt{1-C^{2}(\rho)}\right) .
$$

More can be determined. A simple calculation shows that

$$
\left.\left.D^{2}(|\psi\rangle\rangle\right) \stackrel{\text { def }}{=} D_{1 \mid 23}^{2}(|\psi\rangle\rangle\right)=\frac{1}{2}\left[1-\sqrt{1-4 \operatorname{det}\left(\psi \psi^{\dagger}\right)}\right]
$$

and that

$$
\operatorname{det}\left(\psi \psi^{\dagger}\right)=\frac{1}{2}\left(|a|^{2}+|b|^{2}+2|c|^{2}\right)
$$

these three equations imply that

$$
\begin{aligned}
D^{2}(\rho) & =\frac{1}{2}\left[1-\sqrt{1-2\left(\operatorname{det}\left(\psi \psi^{\dagger}\right)-\left|a b-c^{2}\right|\right)}\right] \\
& =\frac{1}{2}\left(1-\sqrt{\left.\left.1-2\left\{D^{2}(|\psi\rangle\rangle\right)\left[1-D^{2}(|\psi\rangle\rangle\right)\right]-\left|a b-c^{2}\right|\right\}}\right) \\
& \leq \frac{1}{2}\left(1-\sqrt{\left.\left.1-2 D^{2}(|\psi\rangle\rangle\right)\left[1-D^{2}(|\psi\rangle\rangle\right)\right]}\right) .
\end{aligned}
$$

The bound given by the last equation is tight for the range of $D(|\psi\rangle\rangle)$ from 0 to $1 / \sqrt{3}$, as can be seen in Figure 1.

The maximum concurrence of $\rho$ is $2 / 3$, corresponding to $D(\rho)=\left[\frac{1}{2}(1-\sqrt{5} / 3)\right]^{1 / 2} \approx 0.35682$. A state that reduces to a density matrix with this maximum degree of entanglement is the 3-qubit W-state $\left|W_{3}\right\rangle=3^{-1 / 2}[0,1,1,0,1,0,0,0]^{T}$. We thank Saikat Guha for noticing that all symmetric 3 -qubit states that generate a reduced density matrix under trace over a single qubit are symmetrypreserving local unitary transformations of this $\left|W_{3}\right\rangle$. 


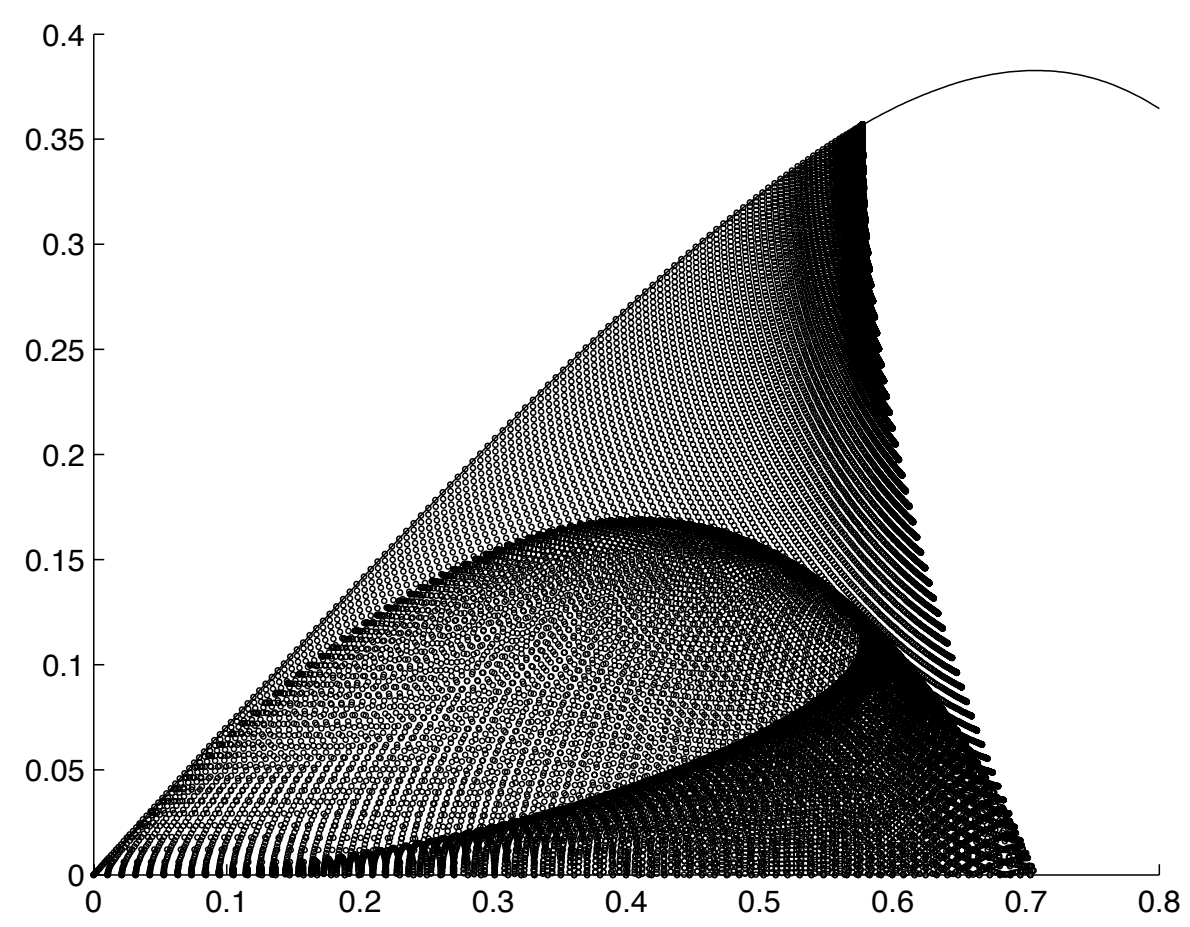

FIG. 1: Scatter plot of possible pairs $[D(|\psi\rangle\rangle), D(\rho)]$ with upper bound given by Eq. (114).

\section{B. One- and two-qubit reductions of bosonic 4-qubit pure states}

We studied 2-qubit reduced density matrices obtained by tracing over two qubits of the maximally "1-3" entangled bosonic 4-qubit pure states $|\psi\rangle$ discussed in Sec. IV.3. These reduced density matrices $\rho \stackrel{\text { def }}{=} \operatorname{tr}_{12}(|\psi\rangle\langle\psi|)$ satisfy the particle-exchange symmetry of Eq. (98). As with the previous case of a 2-qubit mixed state obtained from a 3-qubit pure state, we calculate the degree of entanglement of this 2-qubit density matrix by calculating its concurrence. The condition of being maximally "1-3" entangled implies Eqs. (53)-(55) which in turn imply additional constraints of Eq. (99), and again we have the same properties for the operator $R \stackrel{\text { def }}{=} \rho\left(\sigma_{2} \otimes \sigma_{2}\right) \bar{\rho}\left(\sigma_{2} \otimes \sigma_{2}\right)$ involved in the concurrence so that for the present case Eq. (103) holds. Again $D(\rho)$ then follows from Eq. (12), but now the argument for zero degree of entanglement is more complicated.

The manifold of maximally "1-3" entangled bosonic 4-qubit pure states has dimension $4+2=$ 6 (or one less if overall phase is ignored), so it cannot lie in a single orbit of Bos $L U_{4}$, as is evident from the range of degrees of " $2-2$ " entanglement that can be exhibited by maximally " $1-3$ " entangled states.

Among "1-3" maximally entangled 4-qubit states $|\psi\rangle$ are some whose partial trace over two qubits has a positive degree of entanglement; in the present case, however, we also have to analyze the relation between the "2-2" degree of entanglement and $D(\rho)$. Equation (34) implies that the maximal possible " $2-2$ " split degree of entanglement of any 4-qubit state is $\sqrt{1-1 / 4}=\sqrt{3} / 2$. Equation (32) shows that the maximal "2-2" split degree of entanglement implies $D(\rho)=0$. But because the maximal possible " $1-3$ " split degree of entanglement is $1 / \sqrt{2}<\sqrt{3} / 2$, symmetric maximally entangled 4-qubit states are characterized by the "1-3" split degree $D_{1 \mid 234}^{2}=1 / 2$ to- 
gether with the condition on the " $2-2$ " split degree $D_{12 \mid 34}^{2} \geq 1 / 2$. This condition on the " $2-2$ " split degree falls short of maximal " $2-2$ " entanglement, and so leaves open the question of the value of $D(\rho)$. This question is settled by Sec. IV which implies that the condition $D_{12 \mid 34}^{2} \geq 1 / 2$ is exactly the requirement that $\Lambda_{\max }(\rho) \leq 1 / 2$, which via Eq. (103) implies $C(\rho)=0$. From this via Eq. (12) it follows that no two-qubit density operator $\rho=\operatorname{tr}_{34}(|\psi\rangle\langle\psi|)$ obtained by tracing out two qubits of a 4-qubit symmetric maximally entangled state $\psi$ shows any positive degree of entanglement: $D(\rho)=0$.

As an illustration of the capacity to calculate the degree of entanglement of an 8-by-8 density matrix of rank 2, we calculate all the degrees of entanglement associated with the 4-qubit W-state discussed in [13], where the state is given with phase parameters $\phi, \phi_{1}$, and $\phi_{2}$ as

$$
|W\rangle=\frac{1}{2}\left[|1000\rangle+e^{i \phi_{1}}|0100\rangle+e^{i \phi}\left(|0010\rangle+e^{i \phi_{2}}|0001\rangle\right)\right] .
$$

As given, this state lacks bosonic symmetry; however its orbit under the "big" local transformation group $L U_{4}$ includes the representative bosonic state

$$
\left|W_{0}\right\rangle=\frac{1}{2}(|1000\rangle+|0100\rangle+|0010\rangle+|0001\rangle) .
$$

Because all the degrees of entanglement are invariant under $L U_{4}$, it follows that $D_{1 \mid 234}(|W\rangle)$ $=D_{2 \mid 134}(|W\rangle)=D_{3 \mid 124}(|W\rangle)=D_{4 \mid 123}(|W\rangle)=D_{1 \mid 234}\left(\left|W_{0}\right\rangle\right)$. Similarly all the "22" split degrees of entanglement of $|W\rangle$ are identical to $D_{12 \mid 34}\left(\left|W_{0}\right\rangle\right)$. Further the partial traces of $|W\rangle\langle W|$ are in local orbits of $\left|W_{0}\right\rangle\left\langle W_{0}\right|$ so we have $D_{1 \mid 23}\left(\operatorname{tr}_{1}(|W\rangle\langle W|)=\right.$ $D_{2 \mid 13}\left(\operatorname{tr}_{1}(|W\rangle\langle W|)=D_{1 \mid 23}\left(\operatorname{tr}_{2}(|W\rangle\langle W|)\right.\right.$, etc., all equal to $D_{1 \mid 23}\left(\operatorname{tr}_{1}\left(\left|W_{0}\right\rangle\left\langle W_{0}\right|\right)\right.$ and in the same vein $D\left[\operatorname{tr}_{12}(|W\rangle\langle W|)\right]=D\left[\operatorname{tr}_{23}(|W\rangle\langle W|)\right]$, etc. $=D\left[\operatorname{tr}_{12}\left(\left|W_{0}\right\rangle\left\langle W_{0}\right|\right)\right]$. Thus the whole story is determined from the four quantities

$$
\begin{aligned}
D_{1 \mid 234}(|W\rangle) & =1 / 2, \\
D_{12 \mid 34}(|W\rangle) & =1 / \sqrt{2}, \\
D_{1 \mid 23}\left[\operatorname{tr}_{1}(|W\rangle\langle W|)\right] & =0.3826834, \\
D\left[\operatorname{tr}_{12}(|W\rangle\langle W|)\right] & =\sqrt{1 / 2-\sqrt{3} / 4} \approx 0.2588190 .
\end{aligned}
$$

Equation (119) was calculated following the prescription of Eq. (92). For the W-state, we also have $D(|W\rangle)=\min \left[D_{1 \mid 234}(|W\rangle), D_{12 \mid 34}(|W\rangle)\right]=1 / 2$ which is less than the value $1 / \sqrt{2}$ for a 4-qubit generalized GHZ state.

\section{RESOLVING QUANTUM CHANNELS}

From the standpoint of quantum decision theory joined to scattering theory, the issue of resolving one target from another is this. We assume a radar-like system with a transmitter and a receiver. The receiver knows the possible targets, indexed by $i$, along with the prior probability of each target, denoted $\zeta_{i}$. We take as our measure of resolution the probability of the receiver correctly deciding which target is present at any particular instance, on the basis of whatever information the receiver can extract from an echo scattered by the target from the transmitted electromagnetic field. Unlike communications problems without an echoing target, the issue is not for the receiver to decide what state is transmitted, which it knows, but to decide which of various possible echoes is received, with the idea that the echo indicates something about the target. The echo-as a pure or mixed quantum state-depends on three things: (1) the target, (2) the transmitted state, and 
(3) in a so-called Type-III system, the entanglement between the transmitted state and a reference state held by the transmitter for later comparison with the echo.

Formulating a quantum scattering theory of light to determine echoes available to a quantum ladar awaits another day. One obstacle arises from our expectation that quantum theory, being more general than the classical Maxwell theory, allows at least as much directivity for a light beam as does the classical theory. According to the classical Maxwell theory, the directivity possible for a light beam rises with the allowed ratio of root-mean-square current to radiated power, so that as the ratio increases without bound, so does the directivity, a phenomenon termed supergain [14]. To date, however, quantum optics is limited by simplifying assumptions that rule out analyzing the quantum version of supergain.

Although we must wait for a full theory to get accurate descriptions of scattered echoes, scattering theory appears to fit within the generic framework of quantum channels, which one analyzes as completely positive, trace-preserving (CPT) maps. In this framework, the $i$-th target is expressed by a CPT map $\mathcal{E}_{i}$. The receiver that "knows the possible targets" knows the possible $\mathcal{E}_{i}$, but not which one is present in any particular instance. Framing the issue in terms of quantum channels shows how the entanglement may improve resolution in what is called a Type-III ladar.

A Type-III ladar generates a density matrix $\Gamma$ on a tensor-product Hilbert space $\mathcal{H}_{\text {sto }} \otimes \mathcal{H}_{\text {tran }}$ where $\mathcal{H}_{\text {sto }}$ is the Hilbert space corresponding to storing some of the electromagnetic energy and $\mathcal{H}_{\text {tran }}$ corresponds to energy transmitted. The density matrix $\Gamma$ is entangled across the stored and transmitted parts. Any CPT map $\mathcal{E}_{j}$ folds nicely into a tensor product, which is just what Type-III ladar wants. Let $\boldsymbol{I}_{\text {sto }}$ denote the identity operator on $\mathcal{H}_{\text {sto }}$. The tensor product $\boldsymbol{I}_{\text {sto }} \otimes \mathcal{E}_{j}$ is also a CPT map acting on $\Gamma$ that expresses the scattering of the target concurrent with "doing nothing" to the stored part of $\Gamma$. The best possible Type-III system for given $\mathcal{E}_{i}$ and $\zeta_{i}$ employs the transmitted state $\Gamma$ that maximizes the distinguishability of the echoes, where in case of the $i$-th target, the echo is the density matrix transformed by the channel, namely $\left(1 \otimes \mathcal{E}_{i}\right)(\Gamma)$.

For example, in the simplest case of deciding between two targets that have equal prior probabilities, the receiver decides between $\left(\boldsymbol{I}_{\text {sto }} \otimes \mathcal{E}_{1}\right)(\Gamma)$ and $\left(\boldsymbol{I}_{\text {sto }} \otimes \mathcal{E}_{2}\right)(\Gamma)$. The minimum probability of an incorrect decision given a transmitted state $\Gamma$ on $\mathcal{H}_{\text {sto }} \otimes \mathcal{H}_{\text {tran }}$ is [15]

$$
P_{\operatorname{minErr}}\left(\Gamma, \mathcal{E}_{1}, \mathcal{E}_{2}\right)=\frac{1}{2}\left(1-\frac{1}{2} \operatorname{tr}\left|\left(\boldsymbol{I}_{\text {sto }} \otimes \mathcal{E}_{1}\right)(\Gamma)-\left(\boldsymbol{I}_{\text {sto }} \otimes \mathcal{E}_{2}\right)(\Gamma)\right|\right) .
$$

Although realistic scattering models await the future, toy cases of quantum channels are known in which

$$
\max _{\Gamma} \operatorname{tr}\left|\left(\boldsymbol{I}_{\text {sto }} \otimes \mathcal{E}_{1}\right)(\Gamma)-\left(\boldsymbol{I}_{\text {sto }} \otimes \mathcal{E}_{2}\right)(\Gamma)\right|>\max _{\rho} \operatorname{tr}\left|\mathcal{E}_{1}(\rho)-\mathcal{E}_{2}(\rho)\right|,
$$

which says that the best resolution possible is improved by the stored channel [16]. Moreover the $\Gamma$ that best resolves the scattering processes $\mathcal{E}_{1}$ and $\mathcal{E}_{2}$ is entangled, in some cases maximally [16]. Striking is the finding that the entanglement of $\Gamma$ improves resolution of channels from which the echo shows no entanglement (one speaks of "entanglement-breaking channels"). Even though the echoes are separable, the scattering channels are better resolved than is possible without the TypeIII arrangement "since they live in a higher-dimensional space" [16]. From a mathematical point of view, Type-III ladar systems exploit the peculiarities of positive maps combined with tensor products [17]. Promising work for the future is to analyze quantum channels from the standpoint of scattering theory. 


\section{Acknowledgments}

We thank Jonathan Habif, Zachary Dutton, and Saikat Guha for helpful conversations and valued insights. Thanks to Scott Walck for kindly pointing out the two distinct conventions in the literature by which "local unitary transformations" are defined.

This work was supported in part by Subcontract \#A000039316 between BBN Technologies and Harvard University, under United States Air Force prime contract FA8750-07-C-0206 with Harris Corporation.

\section{Appendix A. DEGREE OF ENTANGLEMENT OF A BIPARTITE PURE STATE}

Because $D(|\psi\rangle)$ is non-negative by definition, we lose no information by studying $[D(|\psi\rangle)]^{2}$ instead of $D(|\psi\rangle)$. Let $\hat{\Phi}$ denote the set of normalized unentangled pure states, so that $|\hat{\phi}\rangle \in \hat{\Phi} \Rightarrow$ $\langle\hat{\phi} \mid \hat{\phi}\rangle=1$. Then for $\alpha$ a complex number,

$$
\begin{aligned}
{[D(|\psi\rangle)]^{2} } & =\min _{\alpha,|\hat{\phi}\rangle \in \hat{\Phi}} \||\psi\rangle-\alpha|\hat{\phi}\rangle \|^{2} \\
& =\min _{\alpha, \hat{\phi}\rangle \in \hat{\Phi}}\left[\langle\psi \mid \psi\rangle-2 \operatorname{Re} \alpha\langle\psi \mid \hat{\phi}\rangle+|\alpha|^{2}\langle\hat{\phi} \mid \hat{\phi}\rangle\right] \\
& =\min _{\alpha, \hat{\phi}\rangle \in \hat{\Phi}}\left[\langle\psi \mid \psi\rangle-2 \operatorname{Re} \alpha\langle\psi \mid \hat{\phi}\rangle+|\alpha|^{2}\right] .
\end{aligned}
$$

With $\alpha$ an undetermined complex number we are free to choose $\langle\psi \mid \hat{\phi}\rangle$ to be real and non-negative:

$$
\langle\psi \mid \hat{\phi}\rangle \geq 0
$$

With this choice, Eq. (A1) becomes

$$
\begin{aligned}
{[D(|\psi\rangle)]^{2} } & =\min _{\alpha,|\hat{\phi}\rangle \in \hat{\Phi}}\left[\langle\psi \mid \psi\rangle-2\langle\psi \mid \hat{\phi}\rangle \operatorname{Re} \alpha+|\alpha|^{2}\right] \\
& =\min _{\alpha,|\hat{\phi}\rangle \in \hat{\Phi}}\left[\langle\psi \mid \psi\rangle-2\langle\psi \mid \hat{\phi}\rangle \operatorname{Re} \alpha+(\operatorname{Re} \alpha)^{2}+(\operatorname{Im} \alpha)^{2}\right]
\end{aligned}
$$

from which it is apparent that the $\alpha$ minimizing the right-hand side satisfies

$$
\operatorname{Im} \alpha=0 \quad \text { and } \quad \alpha \geq 0 .
$$

With this condition on $\alpha$, Eq. (A3) becomes

$$
[D(|\psi\rangle)]^{2}=\min _{\alpha,|\hat{\phi}\rangle \in \hat{\Phi}}\left[\langle\psi \mid \psi\rangle-2\langle\psi \mid \hat{\phi}\rangle \alpha+\alpha^{2}\right] .
$$

Since this is a quadratic form in $\alpha$, the minimum with respect to $\alpha$ is reached when

$$
\frac{\partial}{\partial \alpha}[D(|\psi\rangle)]^{2}=0
$$

which implies

$$
-2\langle\psi \mid \hat{\phi}\rangle+2 \alpha=0
$$


hence

We therefore arrive at Eq. (5).

$$
\alpha=\langle\psi \mid \hat{\phi}\rangle
$$

Comparing Eq. (5) with the definition of the geometric measure $E$ for a normalized pure state $|\hat{\psi}\rangle$ in [2] shows that

$$
D^{2}(|\hat{\psi}\rangle)=2 E(|\hat{\psi}\rangle)-E^{2}(|\hat{\psi}\rangle) .
$$

In the cited work, Shimony shows that

$$
E(|\hat{\psi}\rangle)=1-\alpha_{1},
$$

where $\alpha_{1}$ is the largest coefficient in the Schmidt decomposition of $|\hat{\psi}\rangle$; that coefficient is related to $D(|\hat{\psi}\rangle)$ by

$$
D(|\hat{\psi}\rangle)=1-\alpha_{1}^{2}
$$

The next result, analogous to one obtained for a different geometric measure [2], comes as follows. From Eq. (4) it follows, here using * to denote complex conjugation, that

$$
[D(|\psi\rangle)]^{2}=\min _{x_{k}, y_{\ell}} \sum_{k=1}^{m} \sum_{\ell=1}^{n}\left(\psi_{k l}^{*}-x_{k}^{*} y_{\ell}^{*}\right)\left(\psi_{k l}-x_{k} y_{\ell}\right) .
$$

In order to define a product state, we have to impose a normalization condition on one (but not both) of the factors, namely the condition

$$
\sum_{k=1}^{n_{1}}\left|x_{k}\right|^{2}=1
$$

Since the $y_{\ell}$ are independent variables, differentiation with respect to $y_{\ell}^{*}$ gives for the $x_{k}$ and $y_{\ell}$ that achieve the minimum

$$
\sum_{k=1}^{n_{1}} x_{k}^{*}\left(\psi_{k l}-x_{k} y_{\ell}\right)=0 .
$$

In view of Eq. (A13), this implies

$$
y_{\ell}=\sum_{k=1}^{n_{1}} x_{k}^{*} \psi_{k \ell}
$$

With Eq. (2) and Eq. (A15) for $y_{\ell}$, Eq. (A12) becomes

$$
\begin{aligned}
{[D(|\psi\rangle)]^{2}=} & \left.\min _{x_{k}, y_{\ell}} \sum_{k=1}^{m} \sum_{\ell=1}^{n}\left(\psi_{k l}^{*}-x_{k}^{*} y_{\ell}^{*}\right)\left(\psi_{k l}-x_{k} y_{\ell}\right)\right|_{y_{\ell}=\sum x_{k}^{*} \psi_{k \ell}} \\
= & \min _{x_{k}}\left[\sum_{k=1}^{m} \sum_{\ell=1}^{n}\left|\psi_{k \ell}\right|^{2}-\sum_{k=1}^{m} \sum_{\ell=1}^{n} x_{k}^{*} \psi_{k \ell} y_{\ell}^{*}\right. \\
& \left.-\sum_{k=1}^{m} \sum_{\ell=1}^{n} x_{k} \psi_{k \ell}^{*} y_{\ell}+\sum_{\ell=1}^{n}\left|y_{\ell}\right|^{2} \sum_{k=1}^{m}\left|x_{k}\right|^{2}\right]_{y_{\ell}=\sum x_{k}^{*} \psi_{k \ell}} \\
= & \min _{x_{k}}\left[\langle\psi \mid \psi\rangle-\sum_{\ell=1}^{n}\left|y_{\ell}\right|^{2}-\sum_{\ell=1}^{n}\left|y_{\ell}\right|^{2}+\sum_{\ell=1}^{n}\left|y_{\ell}\right|^{2}\right]_{y_{\ell}=\sum x_{k}^{*} \psi_{k \ell}} \\
= & \min _{x_{k}}\left[\langle\psi \mid \psi\rangle-\left.\sum_{\ell=1}^{n}\left|y_{\ell}\right|^{2}\right|_{y_{\ell}=\sum x_{k}^{*} \psi_{k \ell}}\right]
\end{aligned}
$$




$$
\begin{aligned}
& =\min _{x_{k}}\left[\langle\psi \mid \psi\rangle-\sum_{\ell=1}^{n}\left|\sum_{k=1}^{m} x_{k}^{*} \psi_{k \ell}\right|^{2}\right] \\
& =\min _{x_{k}}\left[\langle\psi \mid \psi\rangle-\sum_{k=1}^{m} \sum_{k^{\prime}=1}^{n_{1}} x_{k^{*}}^{*} x_{k^{\prime}} \sum_{\ell=1}^{n} \psi_{k \ell} \psi_{k^{\prime} \ell}\right] \\
& =\langle\psi \mid \psi\rangle-\max _{|\hat{x}\rangle}\left\langle\hat{x}\left|\left[\operatorname{tr}_{2}(|\psi\rangle\langle\psi|)\right]\right| \hat{x}\right\rangle,
\end{aligned}
$$

where $|\hat{x}\rangle$ denotes a unit vector in the first vector space $\mathcal{H}_{1}$

$$
|\hat{x}\rangle=\left[\begin{array}{c}
x_{1} \\
x_{2} \\
\vdots \\
x_{n_{1}}
\end{array}\right]
$$

In Eq. (A17), in case $|\psi\rangle$ is normalized, the expression $\operatorname{tr}_{2}(|\psi\rangle\langle\psi|)$ is just the reduced density matrix obtained by taking a partial trace over the second tensor-product factor in the pure-state density matrix $|\psi\rangle\langle\psi|$. When $|\psi\rangle$ is not normalized, then $\operatorname{tr}_{2}(|\psi\rangle\langle\psi|)$ can be called a "generalized density matrix," meaning a positive matrix but without requiring a unit trace. It follows that

$$
[D(|\psi\rangle)]^{2}=\langle\psi \mid \psi\rangle-\Lambda_{\max }\left[\operatorname{tr}_{2}(|\psi\rangle\langle\psi|)\right] .
$$

As noted earlier, because the eigenvalues of reduced density matrices are the same for tracing over $\mathcal{H}_{1}$ as for tracing over $\mathcal{H}_{2}$ [6], one gets the same result if in Eq. (A19) $\operatorname{tr}_{2}(|\psi\rangle\langle\psi|)$ is replaced by $\operatorname{tr}_{1}(|\psi\rangle\langle\psi|)$.

\section{Appendix B. PARTIAL TRACE NEVER RAISES THE DEGREE OF ENTANGLEMENT OF ANY PURE BOSONIC STATE}

Here we prove Eq. (B18). We start by assembling some needed thought tools and then proceed to deal with special cases until we build up to the general case.

\section{B.1. Norms for rectangular matrices}

We begin by recollecting a few well known facts about rectangular matrices not available in any single reference we can find. The superscript " $\nmid$ "denotes complex conjugate of the matrix transpose. Let $x$ denote a general column vector with components $x_{j}$ in a complex vector space $\mathcal{H}$ of finite complex dimension. Define the vector norm

$$
\|x\| \stackrel{\text { def }}{=}\left(\sum_{j=1}^{\operatorname{dim}_{\mathbb{C}} \mathcal{H}}\left|x_{j}\right|^{2}\right)^{1 / 2} .
$$

Let $A$ be any $m$-by- $n$ rectangular matrix. Define a mapping from rectangular matrices to nonnegative real numbers by

$$
\|A\| \stackrel{\text { def }}{=} \max _{\|x\| \leq 1 ; x \in \mathbb{C}^{n}}\|A x\| .
$$


(Note that $A x$ is a vector in $\mathbb{C}^{m}$. Restricted to fixed $m$ and $n$, this mapping is a vector norm on $m$-by- $n$ rectangular matrices and satisfies the triangle inequality; restricted further to square matrices of fixed dimension, it is a matrix norm and so submultiplicative; however we impose no such restrictions.) Observe that

$$
\begin{aligned}
\|A\|^{2} & =\left(\max _{\|x\| \leq 1 ; x \in \mathbb{C}^{n}}\|A x\|\right)^{2} \\
& =\max _{\|x\| \leq 1 ; x \in \mathbb{C}^{n}}\left(x^{\dagger} A^{\dagger} A x\right)=\Lambda_{\max }\left(A^{\dagger} A\right)=\left\|A^{\dagger} A\right\|,
\end{aligned}
$$

which implies that

$$
\|\bar{A}\|=\left\|A^{\dagger}\right\|=\left\|A^{T}\right\|=\|A\| .
$$

Lemma: Given an $n$-by- $m_{1}$ matrix $A$ and an $n$-by- $m_{2}$ matrix $B$, form the $n$-by- $\left(m_{1}+m_{2}\right)$ matrix by putting $A$ as a block to the left of $B$ and find that

$$
\|[A B]\|^{2}=\Lambda_{\max }\left(A A^{\dagger}+B B^{\dagger}\right)=\left\|A A^{\dagger}+B B^{\dagger}\right\| \leq\|A\|^{2}+\|B\|^{2} .
$$

\section{B.2. Partial traces}

Partial traces are expressed by rectangular matrices made by rearranging elements of $\left|\psi_{n}\right\rangle$. For $0 \leq k \leq n$, define $\left[\psi^{(k, n-k)}\right]$ to be the $2^{k}$-by- $2^{n-k}$ matrix made by assigning the top $2^{n-k}$ elements of the column vector $\left|\psi_{n}\right\rangle$ in order to the first row of $\left[\psi^{(k, n-k)}\right]$, the second string of $2^{n-k}$ elements of $\left|\psi_{n}\right\rangle$ in order to the second row of $\left[\psi^{(k, n-k)}\right]$, and so on. We then observe that the partial trace on the first $k$ qubits, $1 \leq k \leq n$, is given by

$$
\operatorname{tr}_{1 \ldots k}\left(\left|\psi_{n}\right\rangle\left\langle\psi_{n}\right|\right)=\left[\psi^{(k, n-k)}\right]^{T}\left[\bar{\psi}^{(k, n-k)}\right] .
$$

The same form serves to express successive traces. For example, $\operatorname{tr}_{1}\left(\left|\psi_{n}\right\rangle\left\langle\psi_{n}\right|\right)$ which is equal to $\left[\psi^{(1, n-1)}\right]^{T}\left[\bar{\psi}^{(1, n-1)}\right]^{\dagger}$ is a $2^{n-1}$-by- $2^{n-1}$ density matrix of rank $\leq 2$. Its partial trace over its first qubit is a "doubly reduced" $2^{n-2}-$ by- $2^{n-2}$ density matrix given by

$$
\operatorname{tr}_{1}\left[\operatorname{tr}_{1}\left(\left|\psi_{n}\right\rangle\left\langle\psi_{n}\right|\right)\right]=\operatorname{tr}_{12}\left(\left|\psi_{n}\right\rangle\left\langle\psi_{n}\right|\right)=\left[\psi^{(2, n-2)}\right]^{T}\left[\bar{\psi}^{(2, n-2)}\right] .
$$

\section{B.3. Bosonic symmetry}

To this point we made no use of bosonic symmetry; the first important point of bosonic symmetry is that the partial traces depend only on how many qubits are traced over, and not on which qubits are traced over. For example, for any 4-qubit bosonic state $\left|\psi_{4}\right\rangle$, one has the equality $\operatorname{tr}_{2,4}\left(\left|\psi_{4}\right\rangle\left\langle\psi_{4}\right|\right)=\operatorname{tr}_{1,3}\left(\left|\psi_{4}\right\rangle\left\langle\psi_{4}\right|\right)$.

Denote the permutation group on $n$ objects by $S_{n}$. We are interested in the (reducible) representation of $S_{n}$ on $\mathcal{H}^{\otimes n}$ that corresponds to permuting factors of unentangled states, recognizing that unentangled states span all of $\mathcal{H}^{\otimes n}$. We denote this representation also by $S_{n}$. For example, consider the 3 -qubit space $\left(\mathbb{C}^{2}\right)^{\otimes 3}$. The element of $S_{3}$ that is the cyclic permutation $(1,2,3)$ is represented by an 8-by-8 matrix $s$ acting on the 8-element vectors, so that 


$$
s|\psi\rangle=\left[\begin{array}{llllllll}
1 & 0 & 0 & 0 & 0 & 0 & 0 & 0 \\
0 & 0 & 0 & 0 & 1 & 0 & 0 & 0 \\
0 & 1 & 0 & 0 & 0 & 0 & 0 & 0 \\
0 & 0 & 0 & 0 & 0 & 1 & 0 & 0 \\
0 & 0 & 1 & 0 & 0 & 0 & 0 & 0 \\
0 & 0 & 0 & 0 & 0 & 0 & 1 & 0 \\
0 & 0 & 0 & 1 & 0 & 0 & 0 & 0 \\
0 & 0 & 0 & 0 & 0 & 0 & 0 & 1
\end{array}\right]\left[\begin{array}{l}
\psi_{000} \\
\psi_{001} \\
\psi_{010} \\
\psi_{011} \\
\psi_{100} \\
\psi_{101} \\
\psi_{110} \\
\psi_{111}
\end{array}\right]=\left[\begin{array}{l}
\psi_{000} \\
\psi_{100} \\
\psi_{001} \\
\psi_{101} \\
\psi_{010} \\
\psi_{110} \\
\psi_{011} \\
\psi_{111}
\end{array}\right] .
$$

A pure state $|\psi\rangle$ of $\mathcal{H}^{\otimes n}$ exhibits bosonic symmetry if and only if

$$
\left(\forall s \in S_{n}\right) \quad s|\psi\rangle=|\psi\rangle,
$$

while a mixed state $\rho$ exhibits bosonic symmetry if and only if

$$
\left(\forall s \in S_{n}\right) \quad s \rho s^{\dagger}=\rho .
$$

(Note $s^{\dagger}=s^{T}$.) This leads to the following patterns of indices illustrated for a pure 5-qubit bosonic state in relation to its first two partial traces:

$$
\begin{aligned}
& \left|\psi_{5}\right\rangle^{T}=\left[\psi^{(0,5)}\right] \sim\left[\begin{array}{lll}
0 & 1 & 121223122323341223233423343445
\end{array}\right], \\
& {\left[\psi^{(1,4)}\right] \sim\left[\begin{array}{llllllllllllllll}
0 & 1 & 1 & 2 & 1 & 2 & 2 & 3 & 1 & 2 & 2 & 3 & 2 & 3 & 3 & 4 \\
1 & 2 & 2 & 3 & 2 & 3 & 3 & 4 & 2 & 3 & 3 & 4 & 3 & 4 & 4 & 5
\end{array}\right]} \\
& {\left[\psi^{(2,3)}\right] \sim\left[\begin{array}{llllllll}
0 & 1 & 1 & 2 & 1 & 2 & 2 & 3 \\
1 & 2 & 2 & 3 & 2 & 3 & 3 & 4 \\
1 & 2 & 2 & 3 & 2 & 3 & 3 & 4 \\
2 & 3 & 3 & 4 & 3 & 4 & 4 & 5
\end{array}\right] .}
\end{aligned}
$$

We remark that the decomposition of a bosonic density matrix into pure states can involve pure states that fail to satisfy Eq. (B9). For example if $s|\psi\rangle= \pm|\psi\rangle$, then $s|\psi\rangle\left\langle\psi\left|s^{\dagger}=\right| \psi\right\rangle\langle\psi|$; however in cases we have checked, any such state $|\psi\rangle$ is in a local bosonic orbit of a bosonic state, so we do not worry about this "fermionic" case.

\section{B.4. Proof that the partial trace cannot raise the degree of entanglement of a pure bosonic state}

To introduce the first two ideas needed we prove the simplest special case, namely that for a 3-qubit bosonic state $\left|\psi_{3}\right\rangle$,

$$
D\left(\operatorname{tr}_{1}\left(\left|\psi_{3}\right\rangle\left\langle\psi_{3}\right|\right)\right) \leq D\left(\left|\psi_{3}\right\rangle\right)
$$

To see how bosonic symmetry is crucial, we start without the assumption of bosonic symmetry, and then bring in the assumption of symmetry only when we need it. Without any assumption of bosonic symmetry, let $\left|\psi_{3}\right\rangle \stackrel{\text { def }}{=}\left[c_{1}, c_{2}, c_{3}, c_{4}, c_{5}, c_{6}, c_{7}, c_{8}\right]^{T}$ with $\sum_{j}\left|c_{j}\right|^{2}=1$. Taking the partial trace on the first qubit yields

$$
\begin{aligned}
\operatorname{tr}_{1}\left(\left|\psi_{3}\right\rangle\left\langle\psi_{3}\right|\right) & =\left[\begin{array}{ll}
c_{1} & c_{5} \\
c_{2} & c_{6} \\
c_{3} & c_{7} \\
c_{4} & c_{8}
\end{array}\right]\left[\begin{array}{llll}
\bar{c}_{1} & \bar{c}_{2} & \bar{c}_{3} & \bar{c}_{4} \\
\bar{c}_{5} & \bar{c}_{6} & \bar{c}_{7} & \bar{c}_{8}
\end{array}\right] \\
& =\left[c_{1}, c_{2}, c_{3}, c_{4}\right]^{T}\left[\bar{c}_{1}, \bar{c}_{2}, \bar{c}_{3}, \bar{c}_{4}\right]+\left[c_{5}, c_{6}, c_{7}, c_{8}\right]^{T}\left[\bar{c}_{5}, \bar{c}_{6}, \bar{c}_{7}, \bar{c}_{8}\right]
\end{aligned}
$$


Computing degrees of entanglement one finds

$$
D^{2}\left(\left|\psi_{3}\right\rangle\right)=1-\left\|\left[\begin{array}{llll}
c_{1} & c_{2} & c_{3} & c_{4} \\
c_{5} & c_{6} & c_{7} & c_{8}
\end{array}\right]\right\|^{2}
$$

while

$$
\begin{aligned}
D^{2}\left(\operatorname{tr}_{1}\left(\left|\psi_{3}\right\rangle\left\langle\psi_{3}\right|\right)\right) & \leq 1-D^{2}\left(\left[c_{1}, c_{2}, c_{3}, c_{4}\right]^{T}\right)-D^{2}\left(\left[c_{5}, c_{6}, c_{7}, c_{8}\right]^{T}\right) \\
& =1-\left\|\left[\begin{array}{ll}
c_{1} & c_{2} \\
c_{3} & c_{4}
\end{array}\right]\right\|^{2}-\left\|\left[\begin{array}{ll}
c_{5} & c_{6} \\
c_{7} & c_{8}
\end{array}\right]\right\|^{2} \|^{2} .
\end{aligned}
$$

Without bosonic symmetry there is nothing general to say about the quantity $D^{2}\left(\left|\psi_{3}\right\rangle\right)$ in comparison with $D^{2}\left(\operatorname{tr}_{1}\left(\left|\psi_{3}\right\rangle\left\langle\psi_{3}\right|\right)\right)$; however bosonic symmetry implies that $c_{3}=c_{5}$ and $c_{4}=c_{6}$, with the result that Lemma (B5) applies, so that Eq. (B14) holds. (Actually a slightly weaker symmetry condition suffices, namely that for some real $\theta$, we have $c_{3}=e^{i \theta} c_{5}$ and $c_{4}=e^{i \theta} c_{6}$.)

The characteristic feature of bosonic symmetry is this: To produce $\left[\psi^{(k+1, n-k-1)}\right]$ from $\left[\psi^{(k, n-k)}\right]$, split the top row of $\left[\psi^{(k, n-k)}\right]$ in two and put the right-hand piece immediately below the left-hand piece; work this way down the remaining rows. The result is the same as splitting the whole matrix into two horizontal blocks and making a new matrix by placing the right-hand block beneath the left-hand block, and this is what makes Lemma (B5) applicable. (Equations (B11)-(B13) illustrate a general pattern of indices for $\left[\psi^{(k, n-k)}\right]$, showing that all square subblocks obtained by partitioning into $2^{\ell}$-by- $2^{\ell}$ blocks starting at the upper left corner are symmetric under transpose.)

There is nothing special about qubits; the same procedure works for factor spaces of any finite dimension, so that for any bosonic pure state $|\psi\rangle$, we have

$$
D\left[\operatorname{tr}_{1}(|\psi\rangle\langle\psi|)\right] \leq D(|\psi\rangle)
$$


[1] M. Born, “Zur Quantenmechanik der Stoßvorgänge,” Zeitschrift für Physik 37, 863-867 (1926).

[2] A. Shimony, "Degree of entanglement," Ann. N. Y. Acad. Sci. 755, 675-679 (1995).

[3] F. H. Madjid and J. M. Myers, "Matched detectors as definers of force," Annals of Physics (NY) 319, 251-273 (2005).

[4] T.-C. Wei and P. M. Goldbart, "Geometric measure of entanglement and applications to bipartite and multipartite quantum states," Phys. Rev. A 68, 042307(12) (2003).

[5] For a pure bipartite state, $|\hat{\psi}\rangle$ normalized so that $\langle\hat{\psi} \mid \hat{\psi}\rangle=1$, our $D^{2}(|\hat{\psi}\rangle)$ is equal to $E_{\sin ^{2}}(|\hat{\psi}\rangle)$ in [4]; however, our definition applies also to unnormalized states; note also that the symbol $\Lambda_{\max }$ is given a different meaning in [4] from that assigned in this paper.

[6] M. A. Nielsen and I. L. Chuang, Quantum Computation and Quantum Information (Cambridge University Press, Cambridge, 2000).

[7] W. K. Wootters, "Entanglement of formation of an arbitrary state of two qubits," Phys. Rev. Lett. 80, 2245-2248 (1998).

[8] A. Acin, A. Andrianov, L. Costa, E. Jané, J. I. Latorre, and R. Tarrach, "Generalized Schmidt decomposition and classification of three-quantum-bit states," Phys. Rev. Lett. 85, 1560-1563 (2000); V. Coffman, J. Kundu, and W. K. Wootters, "Distributed entanglement," Phys. Rev. A 61, 052306(5) (2000); S. N. Walck, J. K. Glasbrenner, M. H. Lochman, and S. A. Hilbert, "Topology of the threequbit space of entanglement types," Phys. Rev. A 72, 052324 (2005).

[9] G. M. D'Ariano, P. Lo Presti, and M. F. Sacchi, "Bell measurements and observables," Phys. Lett. A 272, 32-38 (2000).

[10] R. A. Horn and C. R. Johnson, Matrix Analysis (Cambridge University Press, Cambridge, 1985).

[11] P. A. M. Dirac, Quantum Mechanics, 4th ed. (Clarendon Press, Oxford, 1958), Sec. 43.

[12] N. Hadjisavvas, "Properties of mixtures on non-orthogonal states," Lett. Math. Phys. 5, 327-332 (1981); L. P. Hughston, R. Jozsa, and W. K. Wootters, "A complete classification of quantum ensembles having a given density matrix," Phys. Lett. A 183, 14-18 (1993).

[13] S. B. Papp, K. S. Choi, H. Deng, P. Lougovski, S. J. van Enk, and H. J. Kimble, "Characterization of multipartite entanglement for one photon shared among four optical modes," Science 324, 764-768 (2009).

[14] D. Margetis, G. Fikioris, J. M. Myers, and T. T. Wu, "Highly directive current distributions: General theory," Phys. Rev. E 58, 2531-2547 (1998).

[15] C. W. Helstrom, Quantum Detection and Estimation Theory (Academic Press, New York, 1976).

[16] M. F. Sacchi, "Optimal discrimination of quantum operations," Phys. Rev. A 71, 062340(4) (2005); M. F. Sacchi, "Entanglement can enhance the distinguishability of entanglement-breaking channels," Phys. Rev. A 72, 014305(2) (2005); S. Lloyd, "Enhanced sensitivity of photodetection via quantum illumination," Science 321, 1463-1465 (2008); S.-H. Tan, B. I. Erkmen, V. Giovannetti, S. Guha, S. Lloyd, L. Maccone, S. Pirandola, J. H. Shapiro, "Quantum illumination with Gaussian states," Phys. Rev. Lett. 101, 253601(4) (2008).

[17] V. Paulsen, Completely Bounded Maps and Operator Algebras (Cambridge University Press, Cambridge, 2002). As a first pointer into the peculiarities of positive maps, a tensor product with the identity takes the positive map "transpose" to $1 \otimes$ "transpose" which is non-positive. 